\title{
Mammalian nucleolar protein DCAF13 is essential for ovarian follicle maintenance and oocyte growth by mediating rRNA processing
}

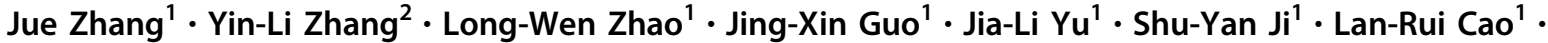 \\ Song-Ying Zhang ${ }^{2} \cdot \mathrm{Li}_{\text {Shen }}{ }^{1} \cdot$ Xiang-Hong Ou ${ }^{3} \cdot$ Heng-Yu Fan $\mathbb{D}^{1,2}$
}

Received: 27 May 2018 / Revised: 22 August 2018 / Accepted: 5 September 2018 / Published online: 3 October 2018

(c) ADMC Associazione Differenziamento e Morte Cellulare 2018

\begin{abstract}
During mammalian oocyte growth, chromatin configuration transition from the nonsurrounded nucleolus (NSN) to surrounded nucleolus (SN) type plays a key role in the regulation of gene expression and acquisition of meiotic and developmental competence by the oocyte. Nonetheless, the mechanism underlying chromatin configuration maturation in oocytes is poorly understood. Here we show that nucleolar protein DCAF13 is an important component of the ribosomal RNA (rRNA)-processing complex and is essential for oocyte NSN-SN transition in mice. A conditional knockout of Dcafl 3 in oocytes led to the arrest of oocyte development in the NSN configuration, follicular atresia, premature ovarian failure, and female sterility. The DCAF13 deficiency resulted in pre-rRNA accumulation in oocytes, whereas the total mRNA level was not altered. Further exploration showed that DCAF13 participated in the 18S rRNA processing in growing oocytes. The lack of 18S rRNA because of DCAF13 deletion caused a ribosome assembly disorder and then reduced global protein synthesis. DCAF13 interacted with a protein of the core box C/D ribonucleoprotein, fibrillarin, i.e., a factor of early pre-rRNA processing. When fibrillarin was knocked down in the oocytes from primary follicles, follicle development was inhibited as well, indicating that an rRNA processing defect in the oocyte indeed stunts chromatin configuration transition and follicle development. Taken together, these results elucidated the in vivo function of novel nucleolar protein DCAF13 in maintaining mammalian oogenesis.
\end{abstract}

These authors contributed equally: Jue Zhang, Yin-Li Zhang, LongWen Zhao

Edited by H. Zhang

Electronic supplementary material The online version of this article (https://doi.org/10.1038/s41418-018-0203-7) contains supplementary material, which is available to authorized users.

\section{Heng-Yu Fan}

hyfan@zju.edu.cn

1 Life Sciences Institute, Zhejiang University, 310058 Hangzhou, China

2 Key Laboratory of Reproductive Dysfunction Management of Zhejiang Province, Assisted Reproduction Unit, Department of Obstetrics and Gynecology, Sir Run Run Shaw Hospital, School of Medicine, Zhejiang University, 310016 Hangzhou, China

3 Assisted Reproduction Unit, Second Hospital of Guangdong Province, China Southern Medical University, Guangzhou, China

\section{Introduction}

In mammalian species, the primordial follicle pool in postnatal ovaries constitutes the oocyte repertory for the entire reproductive life. A small portion of dormant primordial follicles is awakened from time to time to provide a continuous oocyte supply [1]. The awakened oocytes enter an active growth phase that is characterized by remarkable increases in oocyte volume, gene transcription, and protein synthesis [2]. These maternal RNAs and proteins are stored in the germinal vesicle (GV) and ooplasm to support oocyte meiotic maturation and early zygotic development. In fully grown oocytes, RNA transcription is silenced and the chromatin configuration changes from the nonsurrounded nucleolus (NSN) to surrounded nucleolus (SN) type [3].

The best-known factors that maintain normal mammalian oocyte development include a protein complex known as cullin-ring finger ligase 4 (CRL4) [4,5]. An oocyte-specific knockout of the damaged-DNA-binding protein 1 gene (Ddb1), which encodes an essential linker protein of CRL4, causes oocyte growth retardation, follicle atresia, and premature ovarian failure (POF) [4,5]. In Ddbl knockout 
oocytes, genomic DNA is hypermethylated and mRNA transcription of some genes is decreased [5]. Nevertheless, these biochemical changes are not sufficient to explain the severe problems caused by the $D d b 1$ knockout. Particularly, although CRL4 maintains a balanced DNA methylation level by activating the oocyte-enriched DNA demethylase TET3, a Tet3 knockout does not lead to POF [6]. Therefore, the crucial function of CRL4 in the oocyte is likely to be mediated by yet unidentified factors.

There are more than 90 putative CRL4 substrate adapters encoded in a mammalian genome [7]. They are known as DDB1-associated and cullin-4-associated factors (DCAFs) [8]. The in vivo functions of oocyte-derived DCAF1 and DCAF2 in mediating oocyte meiosis and zygotic genome stability have been reported $[9,10]$. By RNA interference (RNAi) screening in Caenorhabditis elegans, we found that a novel DCAF conserved from Schizosaccharomyces pombe to Homo sapiens plays an important part in oogenesis. It is called zk430.7 in C. elegans and its mammalian homolog is called DCAF13. To investigate the in vivo function of DCAF13 during mammalian oogenesis, we studied the influence of a Dcaf13 conditional knockout specific to oocytes.

\section{Results}

\section{RNAi screening for substrate adapters of CRL4 that are essential for oocyte development}

We first investigated which $\mathrm{DCAF}(\mathrm{s})$ was the key substrate adapter of CRL4 for support of female germ cell development. Because it was difficult to systematically evaluate DCAF functions in mammalian oocytes in vivo, we utilized C. elegans, another well-established model system of oogenesis. The C. elegans genome contains 19 putative DCAF-encoding genes that have mammalian homologs (Fig. S1A). Genes encoding CRL4 components and DCAFs were knocked down by a feeding RNAi technique in transgenic worms (pL4440) that express the GFP-tagged histone-72 marker in germ cells and early embryos. RNAi efficiency was confirmed by quantitative RT-PCR analysis of the selected genes (Fig. S1B).

In $C$. elegans hermaphrodites, RNAi depletion of the CRL4 core components encoded by $r b x-1 / 2, c u l-4$, and $d d b$ 1 caused severe oogenesis defects and infertility (Fig. S1C and D). The proximal oocytes were abnormal and no eggs were found in the uterus (Fig. S1D). RNAi of $d c a f-1$ phenocopied the oogenesis defects of CRL4 component depletion (Fig. S1C and D). This observation is in agreement with our previous findings in mouse oocytes [5]. Depletion of $z k 430.7$, which encodes a putative DCAF protein, also caused severe oogenesis defects and infertility (Fig. S1D).

\section{DCAF13 is a nucleolar protein with a dynamic expression pattern during mouse oocyte development}

DCAF13 is a mammalian homolog of the C. elegans $z k 430.7$ gene product. Its biochemical interactions with CUL4-DDB1 have been shown in our recent study [11]. We generated a rabbit monoclonal antibody that recognizes the peptide sequence of GKTVYTGIDHHWKDPV in murine and human DCAF13 proteins. DCAF13 was expressed in oocytes as early as the primordial follicle stage and localized to the nucleolus (Fig. 1a, arrows). After primordial follicle activation, DCAF13 was detected in the nucleolus of growing oocytes with the NSN configuration, reached a peak in oocytes within pre-antral and early antral follicles, and nearly disappeared in the fully grown oocyte with the SN configuration (Fig. 1a, asterisk).

Confocal microscopy results from isolated murine oocytes also showed that DCAF13 is expressed in the nucleolus of growing oocytes with the NSN chromatin configuration, but becomes undetectable in fully grown SN oocytes (Fig. 1b). Furthermore, DCAF13 protein level is lower in fully grown oocytes than in growing oocytes (Fig. 1c). When GFP-tagged DCAF13 and mCherry-tagged nucleolar protein fibrillarin (FBL) were coexpressed in NSN oocytes, these two proteins colocalized to the nucleoli (Fig. 1d, upper panels). Ectopically expressed GFPDCAF13 is capable to localize in the NLB of fully grown SN oocytes as well (Fig. 1d, middle panels). These results suggested that a low protein expression level, but not the inability to localize in NLB, is the reason that DCAF13 was not detected in NLB of SN oocytes.

Similarly, when plasmids expressing these two proteins were cotransfected into HeLa cells, the green and red signals overlapped in the nucleoli (Fig. 1d, lower panels). This distinct and dynamic expression pattern suggested that DCAF13 is a previously unrecognized nucleolar protein and may play an important role in oocyte maintenance and growth (Fig. 1e).

\section{DCAF13 in the oocyte that is required for follicle development and female fertility}

To investigate the in vivo function of DCAF13, we generated a Dcaf13-floxed mouse strain $\left(D c a f 13^{f / f l}\right)$ using a CRISPR/Cas9-based strategy. Exon 2 of Dcafl3 was flanked by 34 bp flox sequences (Fig. 1f). Its deletion $\left(\right.$ Dcaf $13^{-l-}$ ) caused a reading-frame shift in Dcafl3 mRNA. We crossed Dcafl $3^{f l / f l}$ mice with Zp3-Cre and Gdf9-Cre transgenic mice to specifically delete Dcafl3 in oocytes at the primordial follicle and primary follicle stages, respectively (Fig. 1e). Immunohistochemistry (IHC) results showed that DCAF13 is deleted in oocytes starting from the 


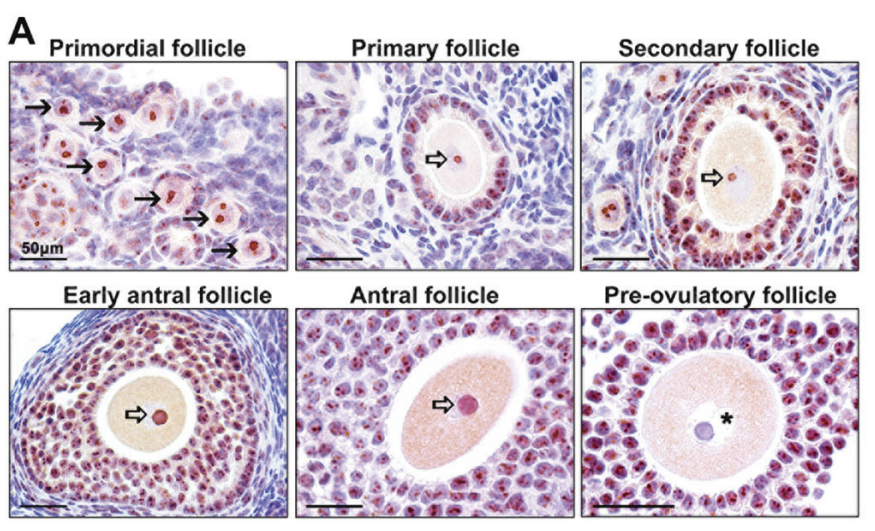

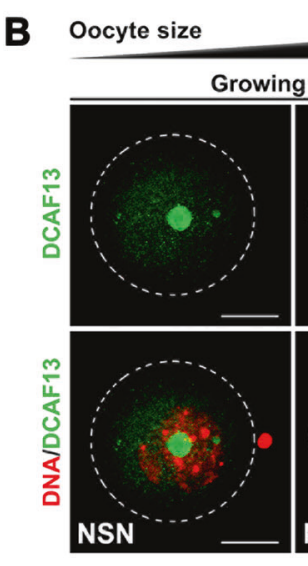

C

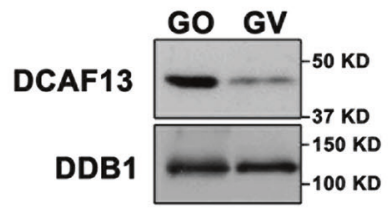

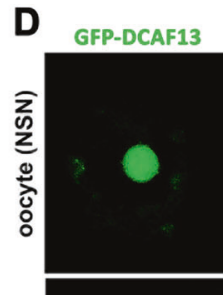
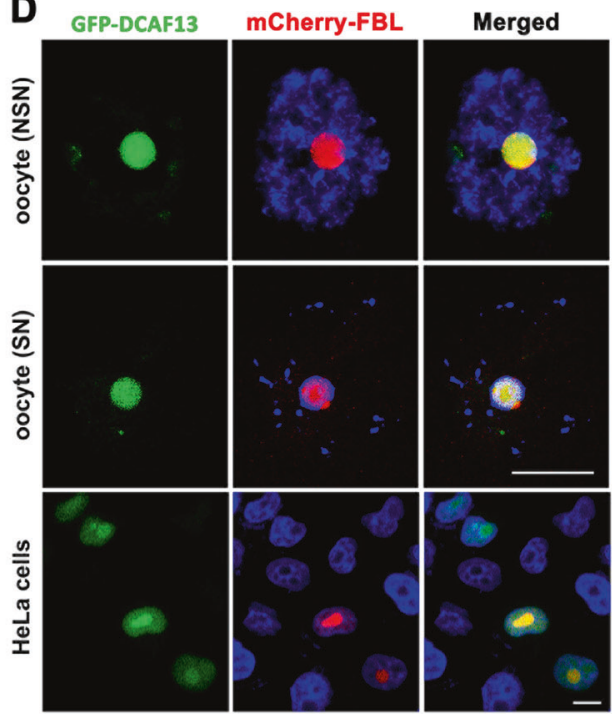

E
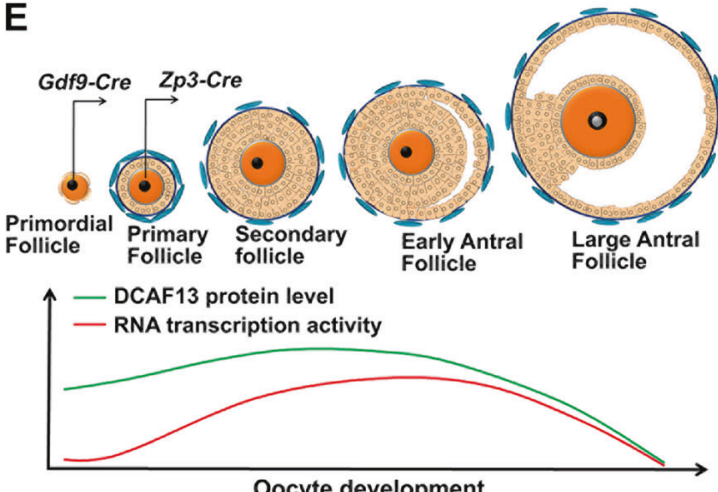

Oocyte development
$\mathbf{F}$

$$
\text { WT -exon1 }
$$

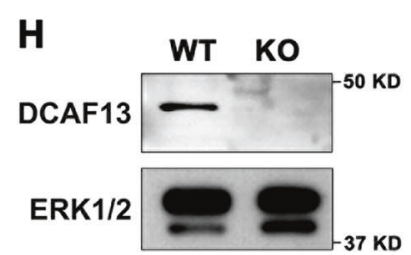

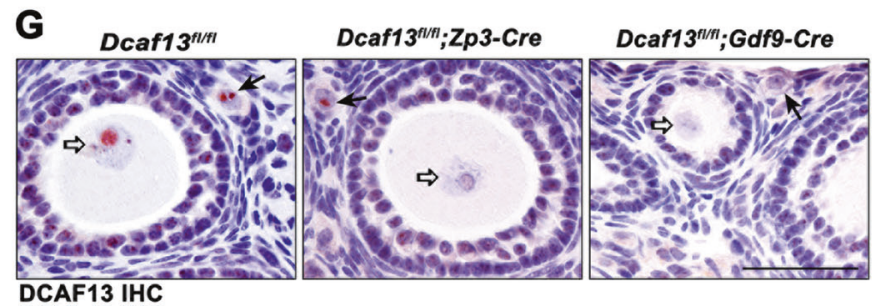

primordial follicle stage in Dcafl $3^{f / f} ; G d f 9-C r e$ mice and from the primary follicle stage in Dcaf $13^{f / f l} ; \mathrm{Zp} 3$-Cre mice (Fig. 1g; arrows indicate primordial follicles). The deletion of the DCAF13 protein in the fully grown oocytes of Dcaf $13^{f l f l} ; \mathrm{Zp} 3$-Cre mice was confirmed by Western blotting (Fig. 1h). 
Fig. 1 Expression and conditional knockout of DCAF13 in growing mouse oocytes. a Immunohistochemistry (IHC) of DCAF13 on ovarian sections showing DCAF13 expression in follicles at indicated developmental stages. Scale bars, $50 \mu \mathrm{m}$. The arrows indicate oocytes in primordial follicles. The hollow arrows indicate the nucleolus of growing oocytes. The asterisk indicates a nucleolus-like body in the fully grown oocyte. b Immunofluorescence (IF) of DCAF13 in growing and fully grown oocytes. DNA was counterstained with 4',6diamidino-2-phenylindole (DAPI). Scale bars, $25 \mu \mathrm{m}$. c Western blot result showing DCAF13 expression in growing oocytes and fully grown oocytes isolated from WT mice at postnatal day 12 and 23, respectively. Total proteins from 100 oocytes were loaded in each lane. d Co-localization of DCAF13 and the nucleolus marker fibrilarin (FBL) in oocytes and in HeLa cells. e A schematic diagram showing ovarian follicular development and oocyte-specific Dcaf13 knockout at primordial and primary follicle stages by Gdf9-Cre and Zp3-Cre, respectively. DCAF13 expression level was high in growing NSN oocytes and decreased in fully grown SN oocytes. Red and green curves represent the level of total RNA transcription activity and level of DCAF13 expression, respectively. f Diagram showing the strategy of Dcafl3 conditional knockout and genotyping approach. The sequences of genotyping primers (GP1, GP2, and GP3) were provided in the Supplementary Table 1. g IHC results showing DCAF13 protein expression in oocytes of mice with indicated genotypes. Arrows and hollow arrows indicate primordial follicles and oocyte nuclei of growing follicles, respectively. $\mathbf{h}$ Western blot results showing DCAF13 levels in 100 fully grown oocytes isolated from control (WT) and Dcafl $3^{f / f}$;Zp3-Cre (KO) mice. ERK1/2 was blotted as a loading control

In a fertility test, 6-week-old Dcafl $3^{f / f l}$;Gdf9-Cre and Dcaf13 ${ }^{f l f l} ; Z$ P 3-Cre female mice ( $n>6$ for each genotype) were crossed with WT males that are known to be fertile for 5 months. Only one pup was born by a Dcafl $3^{f / f} ; \mathrm{Zp3}-\mathrm{Cre}$ female, whereas the other oocyte-specific Dcaf13 knockout females were infertile (Fig. 2a). Hematoxylin and eosin (H\&E) staining of paraffin-embedded ovarian samples indicated that the ovaries of 3-week-old Dcaf1 $3^{f / f l}$; Gdf9-Cre and Dcafl $3^{f / f l}$;Zp3-Cre mice were smaller and had fewer growing follicles than those of the control mice (Dcafl $3^{f / f t}$ littermates; Fig. 2b). Intraperitoneal injection of pregnant mare serum gonadotropin (PMSG) significantly stimulated follicle growth in WT mice, whereas Dcaf13 ${ }^{\text {flfl }}$;Zp3-Cre and Dcaf1 $3^{f / f l}$;Gdf9-Cre mice were unresponsive to PMSG treatment (Fig. 2b). Furthermore, an injection of human chorionic gonadotropin (hCG) at $44 \mathrm{~h}$ after PMSG treatment failed to induce ovulation in Dcafl $3^{f / f l}$;Gdf9-Cre mice (Fig. 2c). Much fewer oocytes were ovulated by Dcafl ${ }^{f l / f}$; Zp3-Cre mice after PMSG and hCG treatments, when compared with their control littermates (Fig. 2c).

The ovaries of 3 -week-old Dcafl $3^{f / f}$;Gdf9-Cre mice (not stimulated by exogenous gonadotropins) contained similar numbers of primordial, primary, and bilayer secondary follicles relative to the control mice, but much fewer secondary follicles with multilayer granulosa cells (Fig. 2d).

The ovaries of 8-week-old Dcafl ${ }^{f / f l}$;Gdf9-Cre and Dcafl $3^{f l / f l}$ ZZp3-Cre mice were significantly smaller than those of WT mice (Fig. 2e) and were devoid of follicles containing more than two layers of granulosa cells and corpora lutea (CLs; Fig. 2b). The ovaries of 8-week-old Dcafl $3^{f / f l}$;Gdf9-Cre mice were deficient in follicles beyond the secondary follicle stage and contained fewer primordial follicles than the control ovaries (Fig. 2f). On the other hand, the Dcafl $3^{f / f t}$;Gdf9-Cre mice had a somewhat greater number of primary follicles in their ovaries at 3 and 8 weeks of age (Fig. 2d and $\mathrm{f}$ ). This result might be caused by a defect of primary-to-secondary follicle transition. At 5 months of age, oocytes disappeared in the ovaries of Dcafl $3^{f / f l}$;Gdf9-Cre mice (Fig. 2g). In comparison, only primordial and primary follicles were seen in the ovaries of 5-month-old Dcafl $3^{f / f f} ; Z p 3-C r e$ mice (Fig. 2b).

Immunohistochemical staining of FOXO1 (a marker of granulosa cells in growing follicles) and MVH (an oocyte marker) in ovarian sections from 5-month-old females further indicated that the Dcafl3 knockout in oocytes of the primordial follicle stage (Dcafl $3^{f / f l} ;$ Gdf9-Cre $)$ caused complete oocyte and follicle loss and resulted in POF, whereas the Dcafl3 knockout in oocytes of the primary follicle stage (Dcaf13 ${ }^{f / f l} ; \mathrm{Zp3}$-Cre) blocked follicle growth beyond this stage (Fig. $3 a$ and $b$ ).

We isolated secondary follicles containing two layers of granulosa cells from 21-day-old control and Dcafl $3^{\text {flff }}$; Gdf9-Cre mice and cultured them in vitro. Control follicles gained multiple layers of granulosa cells on day 4 in the culture and formed a follicle cavity on days 10-12 (Fig. 3c and d). Adding hCG to the culture medium induced follicle rupture and a release of cumulus-oocyte complexes (Fig. 3c). In contrast, the follicles containing Dcafl3 knockout oocytes were arrested at the secondary follicle stage and then degenerated (Fig. 3c and d). None of them developed to the antral follicle stage (Fig. 3d).

\section{DCAF13 deletion inhibits nucleolus maturation in oocytes}

We next analyzed the oocytes isolated from the 3-week-old Dcaf1 $3^{f / f l} ; Z p 3-C r e$ and Dcaf13 ${ }^{f / f l}$;Gdf9-Cre females. The diameters of best-grown Dcaf13-null oocytes were smaller than those of WT (Dcafl $3^{f / f l}$ ) oocytes (Fig. 4a and b). The Dcaf13-null oocytes contained a GV with unclear boundaries and aggregated dark cytoplasmic granules (Fig. 4a). Because the Dcafl $3^{f / f}$;Gdf9-Cre and Dcaf13 ${ }^{f / f l} ; Z p 3-C r e$ mice have similar oocyte phenotypes, we focused on the Dcafl $3^{f / f l}$;Gdf9-Cre mice in the following experiments.

Among the fully grown oocytes isolated from the ovaries of 3-week-old WT mice, approximately three quarters of the cells had the SN chromatin configuration, and one quarter of them had the NSN configuration, as revealed by DAPI staining (Fig. 4c and d). In contrast, this pattern of $\mathrm{SN}$ vs. NSN oocytes was reversed in Dcafl $3^{f / f l}$;Gdf9-Cre mice 
A

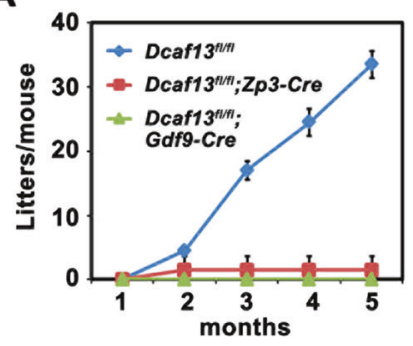

C

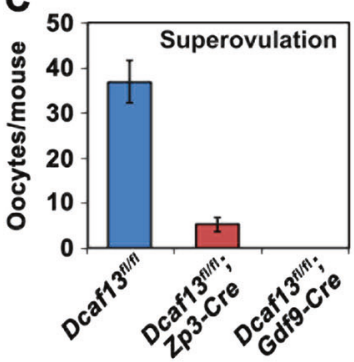

B

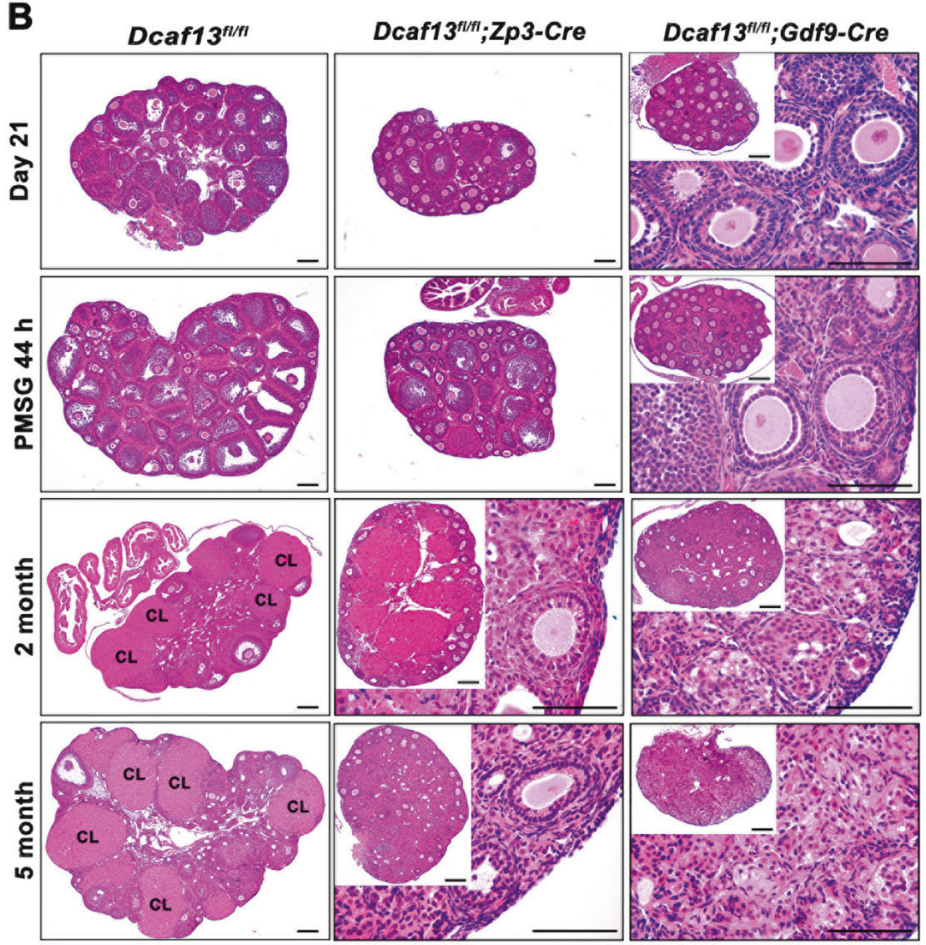

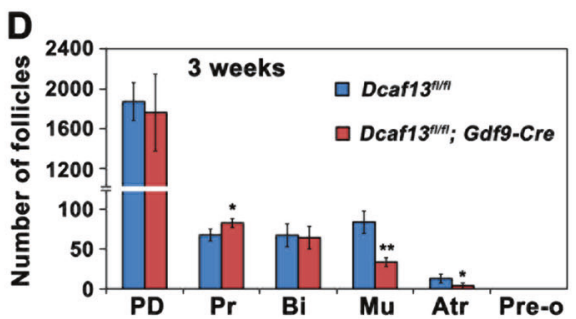

E

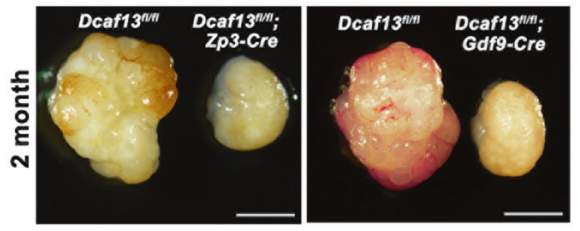

$\mathbf{F}$

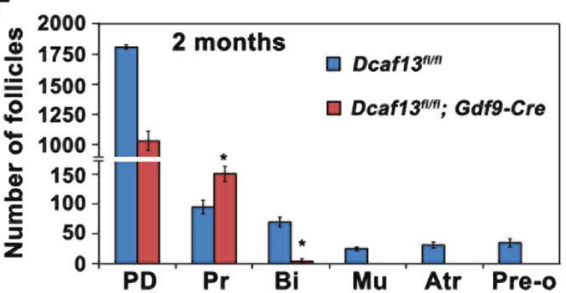

G

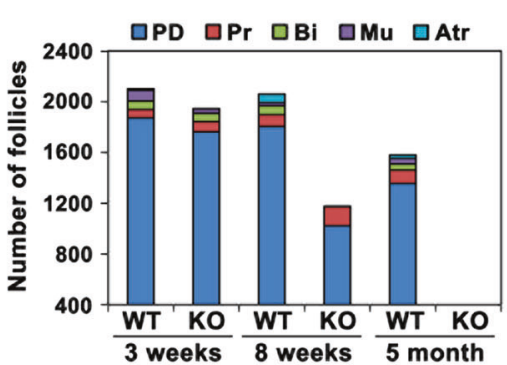

Fig. 2 Phenotype analyses of the oocyte-specific Dcaf13 knockout mice. a Fertility test of female mice. The Dcafl $3^{f / f l}(n=10)$, Dcafl $13^{f /}$ ${ }^{f l} ; \mathrm{Zp3}$-Cre $(n=6)$, and Dcaf1 ${ }^{f l f t} ;$ Gdf9-Cre $(n=5)$ female mice were mated with WT males for 4 months and the total numbers of pups per mice were assessed. Error bars represent SEM. b H\&E staining results showing ovarian histology of 3-week (before and after PMSG treatment), 2-month, and 5-month-old mice with indicated genotypes. Scale bars, $100 \mu \mathrm{m}$. c Numbers of oocytes being ovulated by 3-weekold mice with indicated genotypes after superovulation. Data was expressed mean \pm SEM. $\mathbf{d}$ The number of follicles at indicated stages in the ovaries of 3-week-old Dcafl $3^{f l / f l}$ and Dcafl $3^{f l / f} ;$ Gdf9-Cre mice. Follicles were counted on serial ovarian sections after H\&E staining.

(Fig. 4c and d). This observation indicated that DCAF13 deletion in oocytes inhibited nucleolar maturation.

\section{DCAF13 deletion inhibited protein synthesis, but had little impact on mRNA transcription in oocytes}

Considering that deletion of nucleolar protein DCAF13 may affect global protein synthesis, we incubated growing and fully grown control and Dcaf13-null oocytes (isolated from 12-day-old and 21-day-old mice, respectively) with
Data was shown as mean $\pm \mathrm{SEM}(* P<0.05, * * P<0.01)$. PD primordial follicles, Pr primary follicles, Bi secondary follicles with two layers of granulosa cells, Mu secondary follicles with multiple layers of granulosa cells, Atr antral follicles, Pre-o preovulatory follicles. e Representative ovaries from 2-month-old mice with indicated genotypes. Scale bars, $500 \mu \mathrm{m}$. f The number of follicles at indicated stages in the ovaries of 2-month-old Dcafl $13^{\text {flfl }}$ and Dcaf1 $3^{f / f l} ;$ Gdf9-Cre mice. Follicles were counted on serial ovarian sections after H\&E staining. Data was presented as mean \pm SEM $(* * P<0.01)$. g The number of follicles at indicated stages in the ovaries of 3-week, 8week, and 5-month-old Dcafl $3^{f l / f t}$ and Dcafl $3^{f l / f}$;Gdf9-Cre mice

L-homopropargylglycine (HPG), an analog of methionine that can be detected with the Click-iT cell reaction kit (Life Technologies). In control groups, fully grown SN oocytes had stronger HPG signals than the growing NSN oocytes, indicating that the total protein synthesis level increased during oocyte development (Fig. 4e and f). In comparison, the Dcaf13-null oocytes had weaker HPG signals than those in control oocytes at both developmental stages (Fig. 4e, f).

A 5'-ethynyl uridine (EU) incorporation assay was employed to evaluate the global transcription level in 

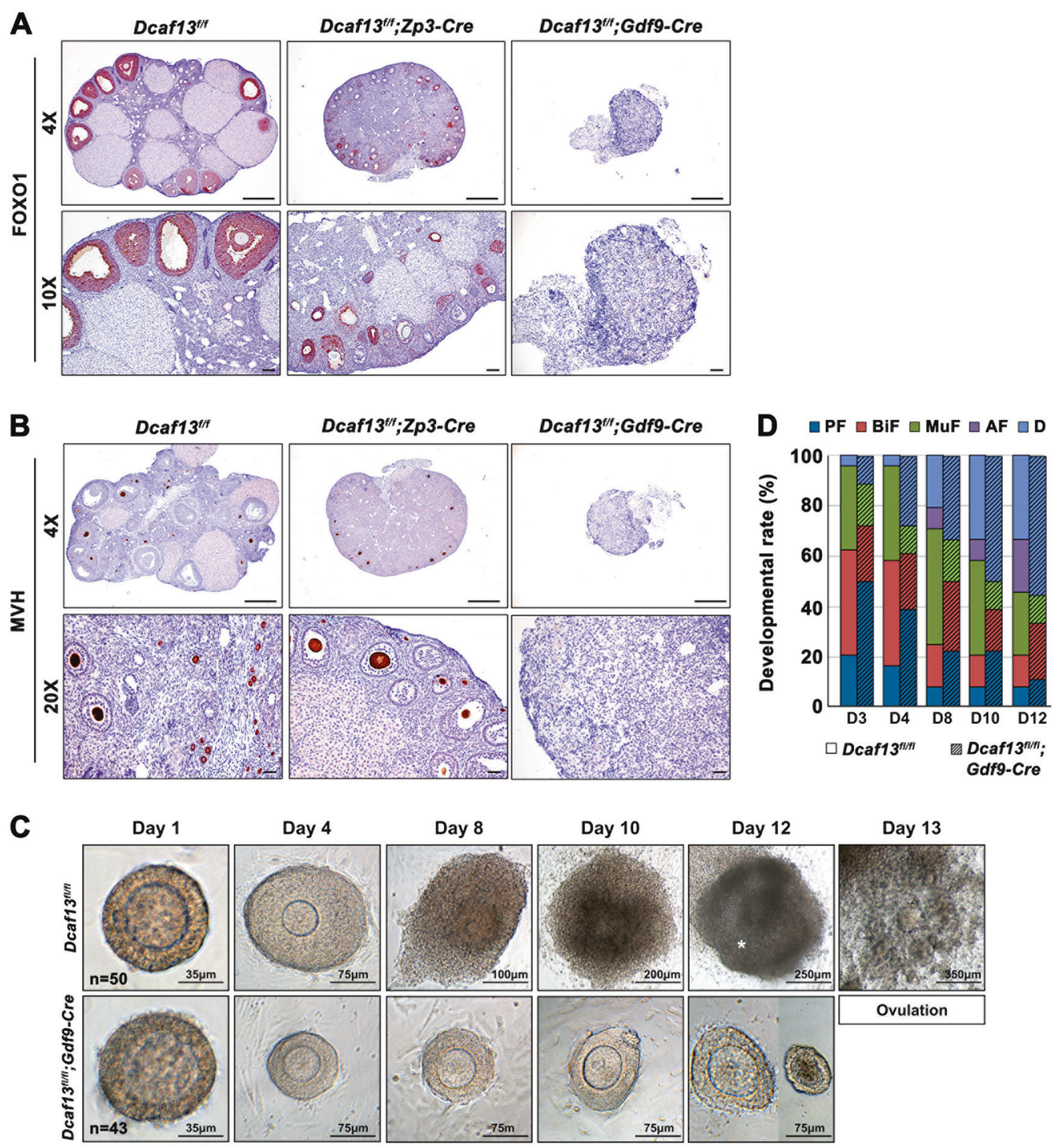

Fig. 3 Dcaf13 knockout in oocyte results in follicular development defect. a and $\mathbf{b}$ Immunohistochemistry staining of forkhead box O1 (FOXO1, a marker of granulosa cells in growing follicles (a)) and mouse VASA homolog (MVH, a germ cell marker (b)) in the ovaries of 5-month-old mice with indicated genotypes. Scale bars, $500 \mu \mathrm{m}$. c In vitro developmental analysis of follicles derived from Dcaf $13^{\text {flft }}$ or Dcaf $13^{f / f l}$; Gdf9-Cre mice. Days in culture were indicated. The asterisk

indicates follicular antrum. Scale bars were shown in each picture. For each genotype, at least 40 follicles were cultured. d Quantification of developmental rates for in vitro cultured follicles in c. PF primary follicles, Bi secondary follicles with two layers of granulosa cells, $\mathrm{MuF}$ secondary follicles with multiple layers of granulosa cells, $\mathrm{AF}$ antral follicles, $d$ degenerated follicles

oocytes. Among the WT oocytes, transcription was active in NSN oocytes but inactive in SN oocytes (Fig. $4 \mathrm{~g}$ ). The proportion of EU-positive oocytes collected from Dcafl $3^{f l f}$; Gdf9-Cre mice was much higher than that in control mice on postnatal day 21 (Fig. 4h). This result is consistent with the observation that the majority of Dcaf13-null oocytes failed to accomplish the NSN-SN transition. Nevertheless, the intensities of EU signals in NSN oocytes derived from control and Dcafl $13^{f / f} ;$; $d f 9$-Cre mice were comparable (Fig. 4g), suggesting that the global transcription activity was not affected after DCAF13 deletion in oocytes.

We carried out RNA sequencing analysis of growing oocytes (from 12-day-old mice) and fully grown oocytes (from 21-day-old mice). The expression levels of genes were assessed by fragments per kilobase of transcript, per million mapped reads (FPKM). We detected 10,917 and 11,028 transcripts in growing oocytes of Dcafl $3^{f / f l}$ and Dcafl $^{\text {fl/fl}}$; Gdf9-Cre mice, respectively, as well as 10,461 and 10,435 transcripts in Dcafl $13^{f l f t}$ and Dcafl $13^{f l f t} ;$ Gdf9-Cre 

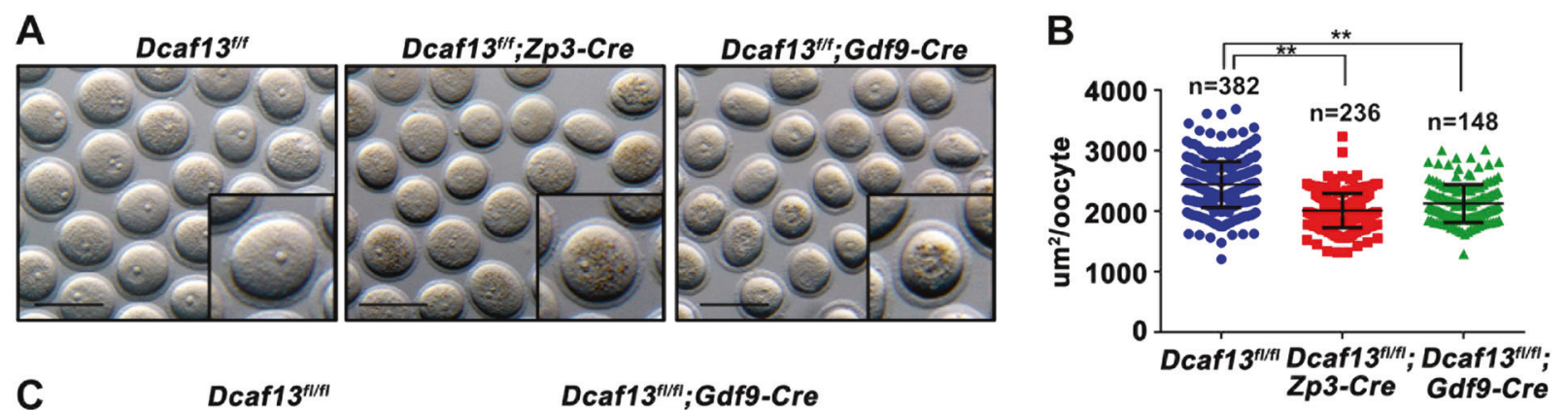

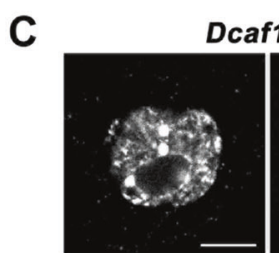

NSN

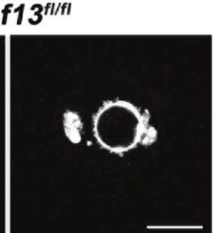

SN

$\mathbf{E}$

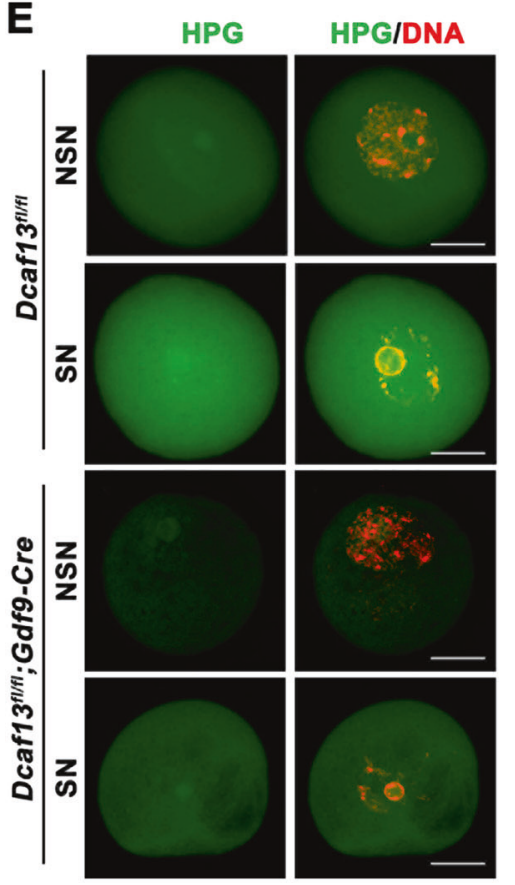

G

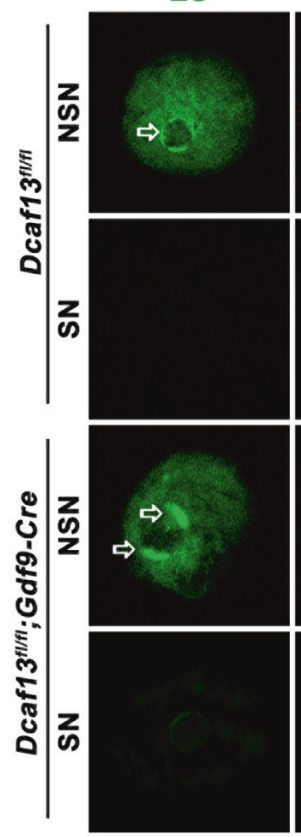

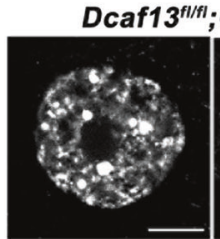

NSN

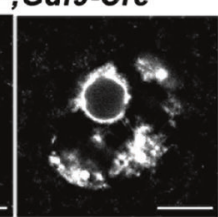

SN

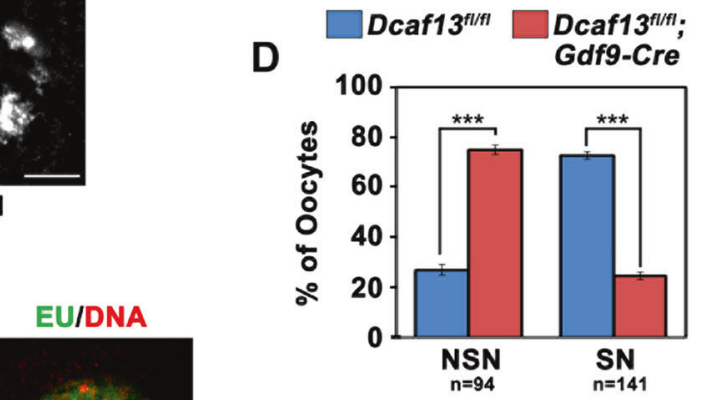

$\mathbf{F}$

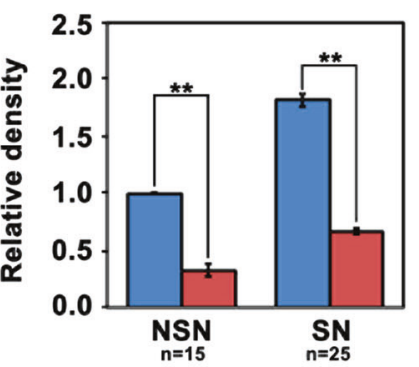

H

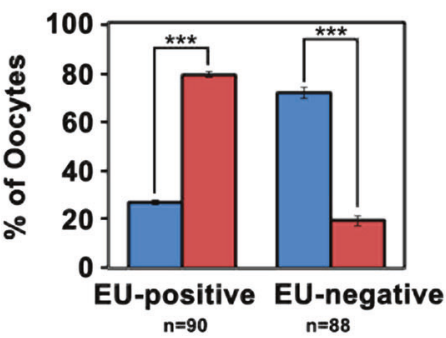

Fig. 4 Dcafl3 knockout inhibits oocyte NSN-SN transition and protein translation. a Germinal vesicle $(\mathrm{GV})$ stage oocytes derived from 21-day-old female mice with indicated genotypes. Scale bars, $100 \mu \mathrm{m}$. b Quantification of oocyte size measured the area of oocytes. Data shown mean \pm SEM $(* * P<0.01)$. c DAPI staining showed the nonsurrounded nucleolus (NSN) and surrounded nucleolus (SN) chromatin configurations in Dcafl $3^{f / f}$ and Dcafl $3^{\text {fl/f }} ;$ Gdf9-Cre oocytes. Scale bars, $25 \mu \mathrm{m}$. d Ratio of NSN-type and SN-type oocytes isolated from 21-day-old Dcafl $3^{f l / f}$ and Dcafl $3^{f l / f} ;$ Gdf9-Cre mice. Data was presented as mean \pm SEM $(* * P<0.01)$. e HPG fluorescent staining results showing protein synthesis activity in NSN-type and SN-type oocytes. Oocytes were isolated from 3-week-old Dcafl $3^{f l / f}$ and Dcafl $3^{f l / t}$;Gdf9-Cre mice and incubated in M2 medium containing $50 \mu \mathrm{M}$ HPG for $1 \mathrm{~h}$ prior to staining. $n=85$ oocytes for each genotype. Scale bars, $50 \mu \mathrm{m}$. f Quantification of HPG signal intensity in e. $n=30$ oocytes in each experimental group. Error bars, SEM $(* * P<0.01)$. g Detection of newly synthesized RNA by EU incorporation in NSN and SN oocytes isolated from the ovaries of 3-week-old Dcaf $13^{f l f}$ and Dcaf $13^{f / f l} ;$ Gdf9-Cre mice. $n=90$ oocytes for each genotype. Scale bars, $50 \mu \mathrm{m}$. h Ratio of EU-positive and EU-negative oocytes in $\mathbf{g}$. Error bars, SEM $(* * * P<0.001)$

Dcaf13-null oocytes are presented in Fig. S2D. Only 0.9\% and $4.7 \%$ of all the detected transcripts were upregulated and downregulated in Dcaf13-null fully grown oocytes, respectively (Fig. S2D). After that, we subdivided the transcripts into five bins by expression level in Dcafl $3^{f / f l}$ fully grown oocytes, with a FPKM level above 1 (Fig. S2A). The four samples showed high correlations $(R \geq$ 0.98; Fig. S2B). There was no significant change in the total mRNA copy numbers in Dcaf13-null oocytes (Fig. S2F). All detected $(\mathrm{FPKM}>1)$ transcriptional changes of genes in 

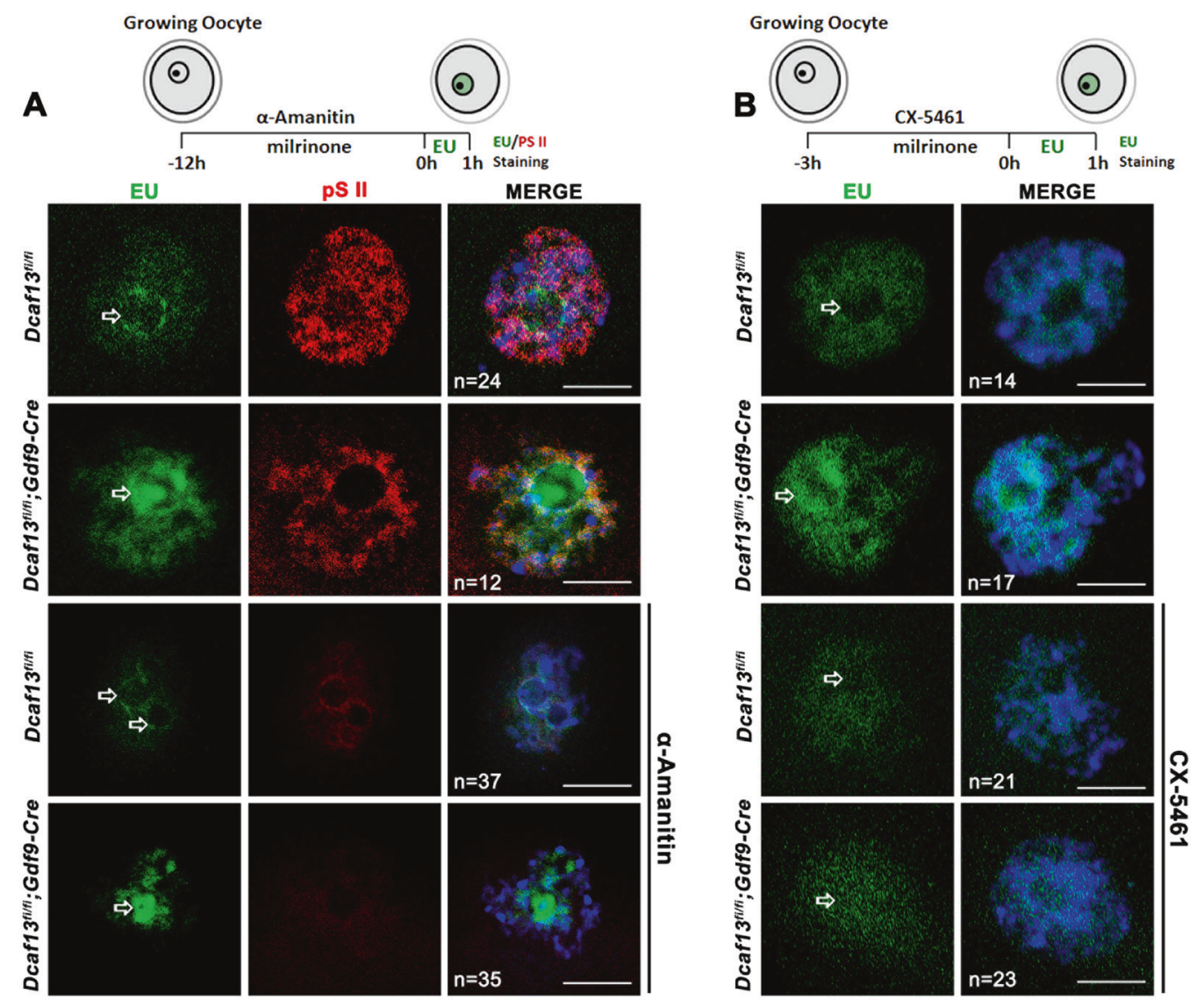

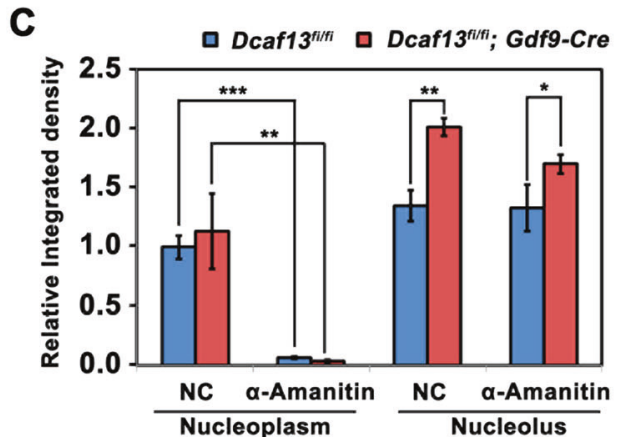

Fig. 5 Detection of mRNA and rRNA biogenesis in growing oocytes. $\mathbf{a}$ and $\mathbf{b}$ EU staining results showing transcription in growing oocytes derived from 12-day-old Dcafl $3^{\text {fl/fl}}$ and Dcaf1 $3^{f l / f} ;$; $d f 9$-Cre mice. Some oocytes were pre-incubated with $10 \mu \mathrm{m}$ RNA polymerase II (Pol II) inhibitor $\alpha$-Amanitin (a) or $10 \mu \mathrm{m}$ RNA polymerase I inhibitor CX5461 (b) before the EU incorporation assay. Phosphorylation of Pol II

fully grown oocytes and found that DCAF13 does not significantly affect gene transcription, regardless of transcript abundance (Fig. S2E).

\section{DCAF13 deletion impaired ribosomal RNA (rRNA) processing}

In the EU incorporation assay, we noticed that more transcripts accumulated in the perinucleolar region of Dcafl3-

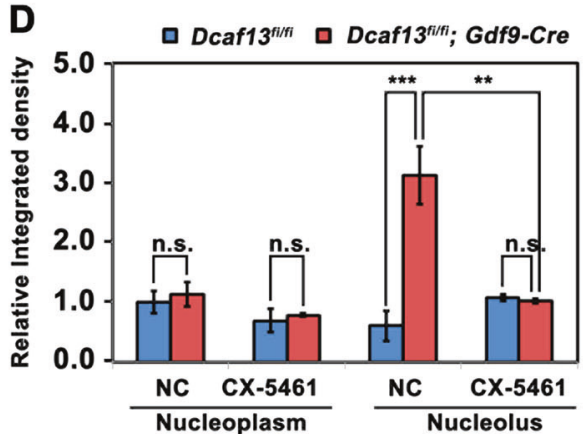

CTD repeat (pS2) was detected by immunofluorescence as an indication of Pol II activity in (a). $n=68$ oocytes in each experimental group. Scale bars, $25 \mu \mathrm{m}$. Arrows indicate nucleoli. c and d Quantification of EU signal intensity in the nucleoplasm and nucleolus from (a) and (b). NC negative control. Error bars, SEM $(* P<0.05$, $* * P<$ $0.01, * * * P<0.001)$

null oocytes relative to control oocytes (Figs. $4 \mathrm{~g}$ and $5 \mathrm{a}$, arrows). When the control and Dcaf13-null growing oocytes were incubated with $\alpha$-Amanitin (which inhibits RNA polymerase II-dependent transcription) for $12 \mathrm{~h}$, the nucleoplasmic transcription signal greatly decreased (Fig. 5a and c). So did the level of RNA polymerase II phosphorylation (pS2), which is a marker of Pol II-mediated transcription (Fig. 5a). In contrast, the newly synthesized $\alpha$ amanitin insensitive transcripts (presumably rRNAs) were 
partially dispersed in GVs of control oocytes but more accumulated in the perinucleolar region of Dcaf13-null oocytes (Fig. 5a and c, arrows). When the WT and Dcaf13null growing oocytes were incubated with CX-5461 (which inhibits RNA polymerase I-dependent transcription) for $3 \mathrm{~h}$, the EU signal in the perinucleolar region was eliminated (Fig. 5b and d), confirming that this signal represented newly transcribed pre-rRNAs. Collectively, these results suggested that rRNA processing activity in growing oocytes was impaired after Dcaf13 deletion.

\section{DCAF13 participates in 18S rRNA processing}

Next, we analyzed the levels of pre-rRNA and rRNA intermediates in oocytes and HeLa cells with or without the Dcafl3 knockout. The scheme of rRNA processing and positioning of probes on processing intermediates in murine and human cells are illustrated in Fig. 6a and S3A. Total RNA samples were extracted from 200 growing oocytes and subjected to northern blotting with digoxigenin-labeled oligonucleotide probes targeting mouse $18 \mathrm{~S}$ and $28 \mathrm{~S}$ rRNAs, as specified in Supplementary Table 1. The targeting positions of probes are presented in Fig. 6a. The northern blot results showed that the level of $18 \mathrm{~S}$ rRNA dramatically decreased in Dcaf13-null oocytes (Fig. 6b).

Fluorescence in situ hybridization (FISH) in oocytes was performed with antisense oligonucleotide probes (FA488$28 \mathrm{~S}$ and Cy3-18S) that matched in the position of mouse $28 \mathrm{~S}$ and $18 \mathrm{~S}$ rRNAs (Fig. 6a). These probes can recognize the localization and amount of $28 \mathrm{~S}$ or $18 \mathrm{~S}$ precursors in the nucleolus and mature $28 \mathrm{~S}$ or $18 \mathrm{~S}$ in the cytoplasm. The results revealed that the hybridization signals were present in the nucleolus of transcriptionally active NSN oocytes (Fig. 6c) but not in the nucleolus of transcriptionally inactive SN oocytes (Fig. S3B), indicating that the signal was specific. Levels of immature $28 \mathrm{~S}$ and $18 \mathrm{~S}$ rRNAs and their precursor in the nucleolus were higher in Dcafl3-null oocytes than in the control oocytes (Fig. 6c-e). The level of mature 18S rRNA (Cy3-18S signal) in the cytoplasm was lower in Dcafl3-null oocytes than in the control oocytes (Fig. 6c, d). By contrast, the level of mature 28S rRNA (FA488-28S signal) in the cytoplasm was higher in Dcaf13null oocytes than in the control oocytes (Fig. 6c-e). These results suggested that Dcafl3 deletion impaired the $18 \mathrm{~S}$ rRNA processing and led to $28 \mathrm{~S}$ rRNA accumulation in Dcaf13-null oocytes.

After that, we extracted total RNA from HeLa cells transfected with siRNAs targeting human Dcaf13 (siDcaf13) and analyzed rRNA processing intermediates by probes targeting $18 \mathrm{~S}$ and $5.8 \mathrm{~S}$ rRNAs (Fig. S3A). The results showed that the DCAF13 protein level decreased in siDcaf13-transfected cells (Fig. S3C). The levels of 26S intermediate pre-rRNA (18S rRNA precursor) and 18S-E
rRNA decreased in the Dcaf13-depleted cells when compared with the control cells (Fig. 6f). The levels of $32 \mathrm{~S}$ intermediate pre-rRNA (28S rRNA precursor) increased in the Dcaf13-depleted cells (Fig. 6f). The level of $12 \mathrm{~S}$ intermediate pre-rRNA (5.8S rRNA precursor) remained unchanged in Dcafl3-depleted cells (Fig. 6f). Then, we performed the RNA-binding protein immunoprecipitation (RIP) experiment on FLAG-tagged DCAF13-expressing HeLa cells. The result suggested that DCAF13 interacted with 18S rRNA (Fig. 6g).

In addition, the polysome profile analysis of HeLa cells indicated that the 40S ribosomal peaks decreased and $60 \mathrm{~S}$ ribosomal peaks increased in siDcafl3-transfected cells, when compared with the control cells (Fig. 6h, i). Correspondingly, the $80 \mathrm{~S}$ ribosomal peaks decreased. Taken together, these results indicated that DCAF13 was involved in 18S rRNA processing in both growing oocytes and HeLa cells. When this pathway was inhibited by Dcafl3 depletion, the $47 \mathrm{~S}$ pre-rRNA was preferentially processed into $28 \mathrm{~S}$ rRNA.

\section{The rRNA processing defect during follicle development inhibited NSN-SN transition in the mouse oocyte}

Co-immunoprecipitation results revealed that DCAF13 interacted with fibrillarin and NOP56 (Fig. 7a). The interactions were not weakened by RNase treatment (Fig. 7a). Fibrillarin is expressed in the oocytes of primordial follicles (Fig. 7b, arrows). During primordial follicle activation, fibrillarin became more concentrated in the nucleolus of awakened oocytes (Fig. 7b, black open arrows). This pattern became more pronounced in oocytes of secondary and early antral follicles. Nevertheless, fibrillarin was absent in the NLB of fully grown oocytes (Fig. 7b, red open arrows) and was redistributed to the cytoplasm. Confocal microscopy of isolated oocytes (Fig. 7c) showed that the fibrillarin signal was abundant in the nucleolus of growing oocytes (isolated from 12-day-old mice) and disappeared from the NLB of fully grown oocytes (isolated from 21-day-old mice after PMSG injection). Among the oocytes collected from mouse ovaries on postnatal day 21 , fibrillarin was detected at the boundary of the nucleolus of NSN oocytes; this signal decreased during $\mathrm{NSN}-\mathrm{SN}$ transition and disappeared in $\mathrm{SN}$ oocytes (Fig. 7d).

We knocked down fibrillarin mRNA and protein in growing oocytes by siRNA microinjection (Fig. 7e, f). HPG fluorescent staining showed that the global protein synthesis activity was low in $\mathrm{Fbl}$ siRNA-injected oocytes (Fig. 7f, g). Then, we microinjected $F b l$ siRNAs into oocytes enclosed by bilayer granulosa cells and examined the development of these follicles in vitro. Forty percent of the control follicles developed to the antral follicle stage within 12 days. In 

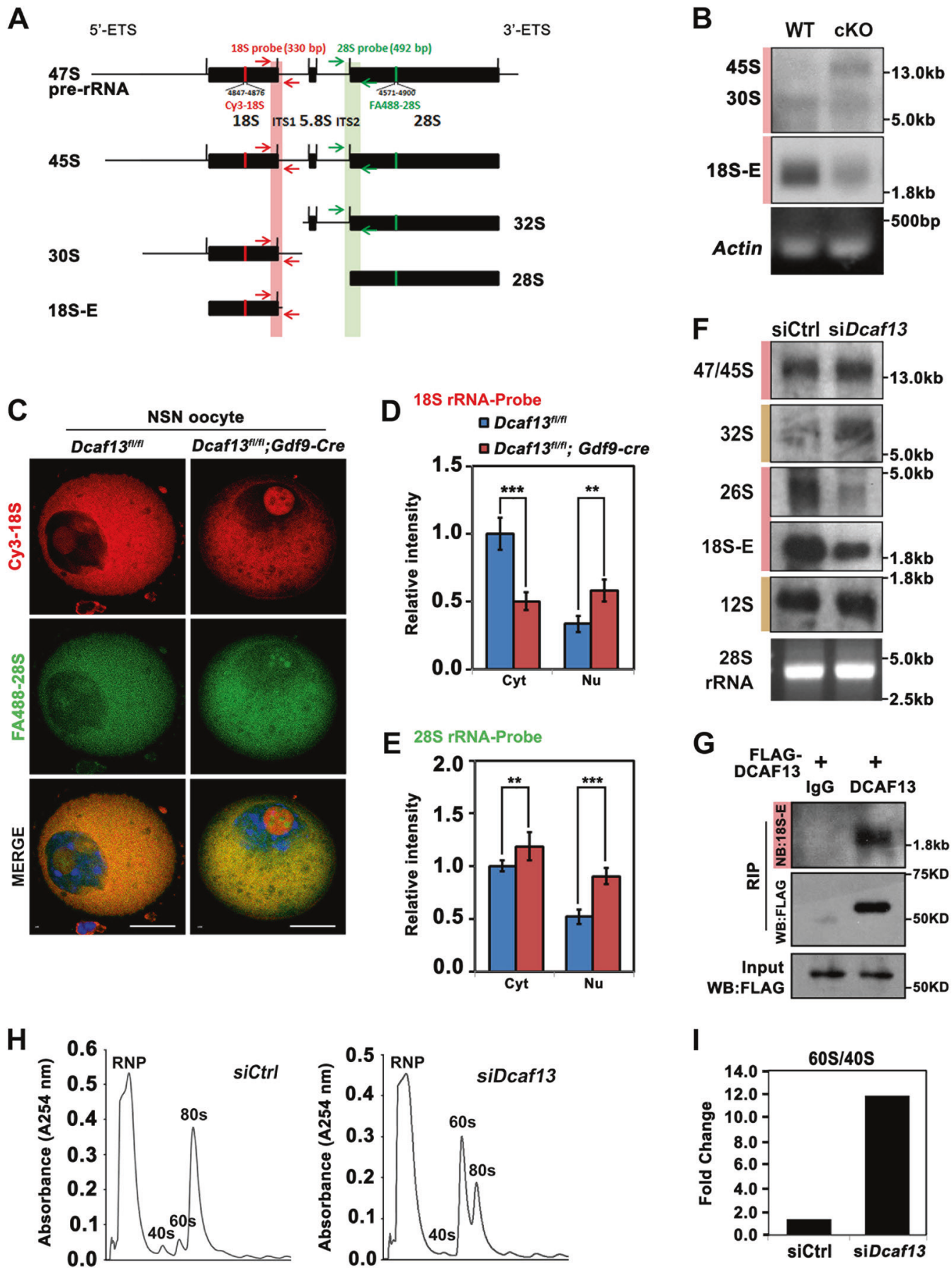

Fig. 6 DCAF13 is involved in rRNA processing in oocytes and HeLa cells. a, b Northern blot of $18 \mathrm{~S}$ rRNA in total RNAs isolated from 200 growing Dcafl $3^{f / f l}$ or Dcafl $3^{f / f l} ; G d f 9$-Cre oocytes. RT-PCR of the Actin was performed as an internal control. c Fluorescent in situ hybridization (FISH) of antisense oligonucleotide probes recognizing the mouse 28S rRNA (Green) and 18S rRNA (Red) in Dcafl $3^{f / f l}$ and Dcaf $13^{f / f} ;$ Gdf9-Cre oocytes. $n=68$ oocytes in each genotype. Scale bars, $25 \mu \mathrm{m}$. d and e Quantification of $28 \mathrm{~S}$ (d) and 18S (e) rRNA signal intensity in the nucleolus or cytoplasm from c. Error bars, SEM $(* P<0.05, * * P<0.01, * * * P<0.001)$. Cyt cytoplasm, $\mathrm{Nu}$ nucleolus. f Northern blot showing rRNA processing intermediates using probes targeting 18S rRNA and 5.8S rRNAs in HeLa cells transfected with
siCtrl or siDcaf13. Pink and brown bars indicated blotting bends by $18 \mathrm{~S}$ and 5.8S rRNA probes, respectively. $\mathrm{g}$ RNA immunoprecipitation (RIP) results showing the interaction of DCAF13 with $18 \mathrm{~S}$ rRNA. HeLa cells were transfected with FLAG-tagged DCAF13 for $48 \mathrm{~h}$ and then subjected to immunoprecipitation with anti-FLAG beads. Protein-RNA complex were extracted for Northern blot of $18 \mathrm{~S}$ rRNA and Western blot of FLAG-DCAF13, respectively. $\mathbf{h}$ The polysome profiles to monitor ribosome assembly in HeLa cells were analyzed by sucrose density gradient centrifugation. The curve graph showing the polysome profiles of HeLa cells transfected with siCtrl or siDcafl3. i The ratio of $60 \mathrm{~S}$ to $40 \mathrm{~S}$ ribosomal peak value in HeLa cells transfected with siCtrl or siDcaf13 
A

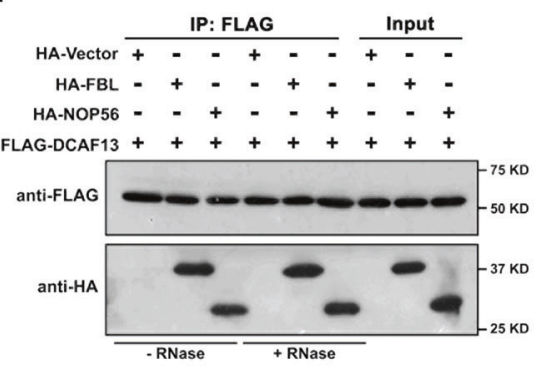

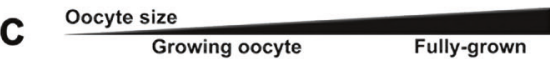

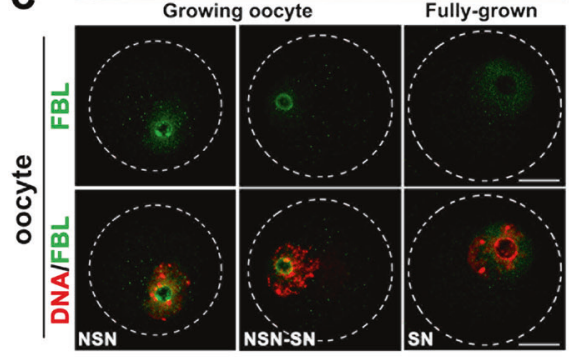

D

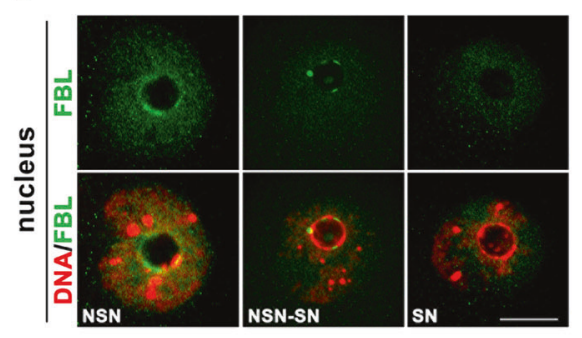

H

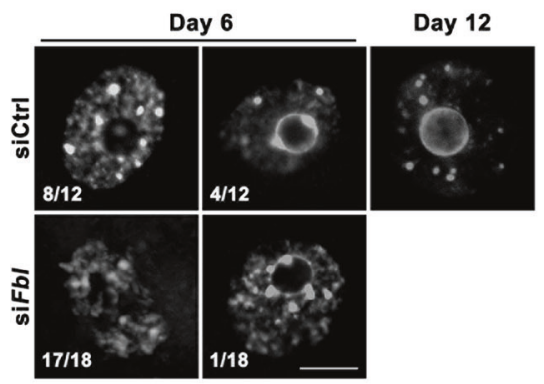

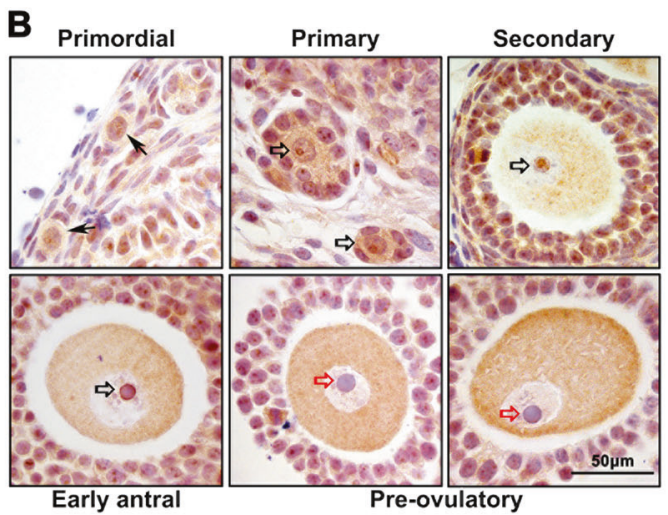
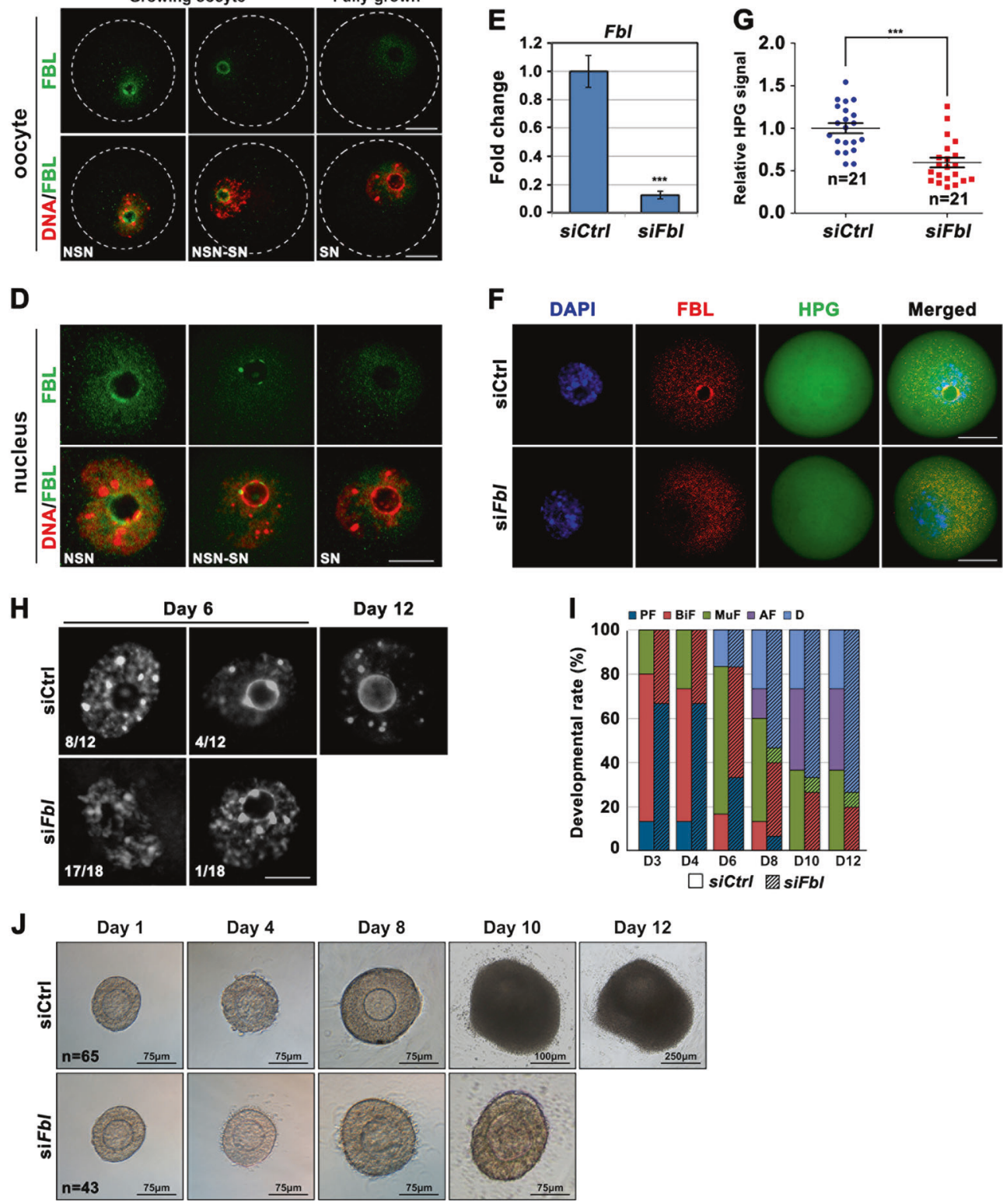

these follicles, $62 \%$ of oocytes contained GVs of the SN configuration (Fig. 7h). On the other hand, a Fbl knockdown in oocytes arrested follicle development at the secondary follicle stage (Fig. 7i, j). Next, we isolated oocytes from the follicles on day 6 of follicle culture after microinjection and examined their chromatin configuration. 
Fig. 7 Depletion of FBL in oocytes caused rRNA processing and follicle development defects. a Co-immunoprecipitation result showing DCAF13 interaction with FBL or NOP56. HeLa cells were cotransfected with Flag-DCAF13 and HA-FBL or HA-NOP56 expression plasmids for $48 \mathrm{~h}$. Target proteins were immunoprecipitated using anti-FLAG beads and subjected to Western blotting with FLAG and HA antibodies. Input cell lysates were immunoblotted with an anti-HA antibody to determine the expressions of FBL and NOP56. b Immunohistochemistry results showing FBL expression and localization in oocytes at the indicated stages. Arrows, black hollow arrows, and red hollow arrows indicate primordial follicles, nucleoli of growing oocytes, and nucleoli of fully grown oocytes, respectively. Scale bars, $50 \mu \mathrm{m}$. c Immunofluorescence of DCAF13 in growing and fully grown oocytes collected from 12-day-old and 21-day-old WT mice, respectively. d Immunofluorescence of DCAF13 in NSN and SN oocytes collected from 21-day-old WT mice. Only the germinal vesicles were shown. Scale bars, $25 \mu \mathrm{m}$. e RT-PCR results showed mRNA levels of $\mathrm{Fbl}$ in oocytes at $24 \mathrm{~h}$ after siCtrl or siFbl microinjection. Error bars, SEM. f-g Quantification (g) and images (f) of HPG signal (green) in oocyte microinjected with siCtrl or siFbl. Growing oocytes were incubated with HPG for $1 \mathrm{~h}$, started at $24 \mathrm{~h}$ after siRNA microinjection. The efficiency of $F b l$ RNA interference was determined by FBL immunofluorescence (red). Scale bars, $50 \mu \mathrm{m}$. Error bars, SEM $(n=21$ oocytes, $* * * P<0.001)$. h DAPI staining showing chromatin configurations in oocytes with or without $\mathrm{Fbl}$ depletion. Primary follicles isolated from 13 to 14-day-old WT mice. Follicle-enclosed oocytes were microinjected with siCtrl or siFbl and were cultured in vitro. The oocytes were isolated from the follicles and stained with DAPI at days 6 and 12 after cultures. Scale bars, $25 \mu \mathrm{m}$. $\mathbf{i}$ The statistics of the culture of the pre-antral follicles in $\mathbf{g}$ in vitro for 12 days. PF primary follicle, BiF bilayer secondary follicle, MuF multilayer secondary follicle, AF antral follicle, D degenerated. j Development and differentiation of a representative follicle from the control-injected or oocyte-injected siRNA targeting $\mathrm{Fbl}$ cultured for 12 days in vitro. Scale bars as shown in every picture. For each sample, at least five females were used

A third (4/12) of oocytes from control follicles had pre-SN GVs. In contrast, all oocytes from $F b l$ siRNA-injected follicles contained NSN GVs (Fig. 7h).

\section{Discussion}

In mice, all oocytes within the pre-antral follicles belong to the NSN type [3]. Only when the follicles grow to the antral stage, do the fully grown GV oocytes transition from the NSN to SN type. It is well known that SN oocytes have better developmental competence than NSN oocytes after meiotic maturation and fertilization $[3,12]$. Nevertheless, the genes and molecules that affect this process in vivo have been poorly investigated [13]. Deletion of the novel nucleolar protein DCAF13 in oocytes here impaired NSN-SN configuration transition, indicating that nucleolar function is crucial for this process. DCAF13 depletion in oocyte resulted in follicular developmental arrest at the secondary follicle stage and caused oocyte death in the long run. Therefore, the level of DCAF13 in a growing oocyte may serve as a key indicator of follicular and oocytic developmental competence (Fig. 8).

The growing oocyte is a translationally active cell and must accumulate high-quality maternally derived proteins to support subsequent embryonic development [14]. Thus, perturbations of ribosomal and protein metabolism are likely to have a profound impact on oocyte health. Prior to mammalian oocyte maturation, growing oocytes go through a transcriptionally and translationally active growth phase, in which necessary RNAs including mRNAs and rRNAs are synthesized and reach a peak of expression at the beginning of follicular antrum formation [15]. In cattle, oocytes of primordial follicles have only a partial nucleolus consisting of the granular component but lacking the fibrillar center and the dense fibrillary component [16]. The functional fibrillogranular nucleolus is re-established in the oocytes of early secondary follicles. This observation and our findings indicate that active nucleolar function is crucial for follicular development beyond the early secondary follicle stage.

Our results indicated that DCAF13 colocalizes and directly interacts with the key nucleolar protein fibrillarin. Nevertheless, how the fibrillarin function is regulated by DCAF13 remains unclear. Fibrillarin-specific complex is directly involved in different post-transcriptional processes, such as pre-rRNA cleavage, rRNA methylation, and ribosome assembly [17]. Methylation of rRNA is carried out in more than 100 sites. FBL is the main methyltransferase for all these sites. DCAF13 may function as a chaperone or scaffold protein in the pre-rRNA processing complex that involves FBL [17]. As an adapter of CRL4 E3 ligase, DCAF13 may also mediate mono-ubiquitination or polyubiquitination of FBL. These post-translational modification of fibrillarin may regulates its activity.

Our present study offers a new concept: nucleolar functional defects and insufficient rRNA biosynthesis are potential reasons for POF. In agreement with our findings, a recent study showed that oocyte proteostasis is disrupted with age, and the gene expression changes are related to the nucleolus [18]. Advanced reproductive age of mouse oocytes was associated with nucleolar structural changes indicative of disrupted functioning. A $56 \mathrm{kDa}$ protein, SOF1, a homolog of DCAF13 in yeast, was found to be located in the nucleolus and to engage in physical association with small nucleolar RNA (snoRNA) U3 and NOP1 (fibrillarin) [19]. Depletion of SOF1 leads to impaired prerRNA processing and inhibition of $18 \mathrm{~S}$ rRNA production. Our experiments on DCAF13-null oocytes and HeLa cells indicated that DCAF13 performs an evolutionarily conserved essential function in 18S rRNA processing and revealed that DCAF13 is a previously unknown component of the nucleolar rRNA processing machinery in mammals. 

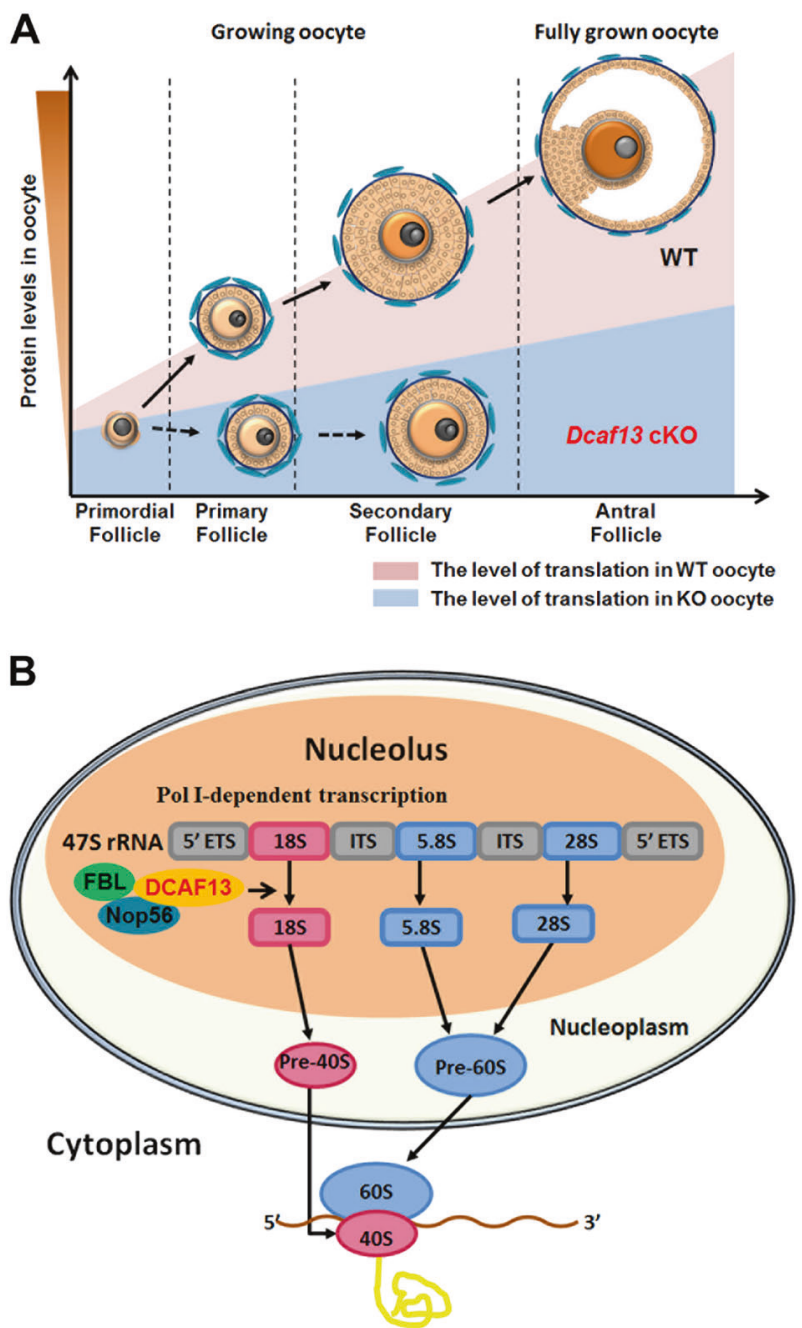

Fig. 8 Summary of DCAF13 function in rRNA processing and oocyte and follicle development. a During follicle development, growing oocytes undergo a translationally active growing phase in which necessary protein are synthesized. DCAF13 deletion in oocytes compromised nucleolus NSN-SN configuration transition and caused global downregulation of protein accumulation, which cause follicular development arrest in early secondary follicles. b In the nucleolus, the 47S ribosomal precursor RNA (pre-rRNA) is cleaved to form the mature $28 \mathrm{~S}, 18 \mathrm{~S}$, and $5.8 \mathrm{~S}$ rRNAs. During this maturation process, the pre-rRNA and its processing intermediates undergo numerous post-transcriptional modifications, which are guided and catalyzed by small nucleolar RNAs (snoRNPs) and their interacting proteins including NOP56 and fibrillarin. DCAF13 has a strong interaction with NOP56 and fibrillarin, which are associated with box C/D U3 snoRNA, to participate in the $18 \mathrm{~S}$ rRNA processing. DCAF13 ensures enough mature 18S rRNA in the cytoplasm to assemble $40 \mathrm{~S}$ subunit of ribosomes and regulates protein translation

\section{Materials and methods}

\section{Animals}

Dcaf13 floxed mice were generated using CRISPR-CAS9 system, as illustrated in Fig. 1c. Gdf9-Cre mice were previously reported [20]. All mouse strains had a C57B6 background. Wild-type C57B6 mice were obtained from the Zhejiang Academy of Medical Science, China. Animal care and experimental procedures were conducted in accordance with the Animal Research Committee guidelines of Zhejiang University.

\section{Oocyte collection and in vitro culture}

Growing oocytes were obtained from the ovaries of 13.5-14.5-day-old Dcaf1 $3^{f / f l}$ and Dcafl $3^{f / f l} ;$ Gdf9-Cre female mice. Fully grown GV oocytes were obtained from the ovaries of 3-week-old female mice $48 \mathrm{~h}$ after intraperitoneal injection of 5 IU of PMSG (Ningbo Sansheng Pharmaceutical Co., Ltd., China). Oocytes were released by puncturing follicles with a fine needle in $37{ }^{\circ} \mathrm{C}$ pre-warmed M2 medium (Sigma-Aldrich).

\section{In vitro follicle culture}

Primary follicles $(50 \mu \mathrm{m}-0.1 \mathrm{~mm}$ in diameter) were isolated from the ovaries of 14-day-old females and were cultured with a-minimal essential medium (a-MEM) supplemented with $5 \%$ fetal bovine serum (FBS) (Invitrogen), $5 \mathrm{mg} / \mathrm{ml}$ insulin, $5 \mathrm{mg} / \mathrm{ml}$ transferrin and $5 \mathrm{ng} / \mathrm{ml}$ selenium (ITS; Sigma, Belgium), and $10 \mathrm{mIU} / \mathrm{ml}$ recombinant $\mathrm{hFSH}$ (Gonal-F, Merck Serono, Germany). The follicles were cultured at $37{ }^{\circ} \mathrm{C}, 100 \%$ humidity, and $5 \% \mathrm{CO}_{2}$ in air. The ovulatory stimulus was given at day 12 by supplementing $1.5 \mathrm{IU} / \mathrm{ml}$ hCG (American Pharmaceutical Partners, Schaumburg, IL, USA) and $5 \mathrm{ng} / \mathrm{ml}$ recombinant epidermal growth factor (rEGF; Boehringer Mannheim, Germany) to the follicle culture medium. At $13 \mathrm{~h}$ after the ovulation stimulus, free-floating cumulus-oocyte complexes were imaged and counted.

\section{Cell culture, plasmids construction, and transfection}

HeLa cells were cultured in Dulbecco's modified Eagle medium (DMEM) supplemented with 10\% FBS, $100 \mathrm{IU} / \mathrm{ml}$ penicillin, and $100 \mathrm{mg} / \mathrm{ml}$ streptomycin at $37{ }^{\circ} \mathrm{C}$ with $5 \%$ $\mathrm{CO}_{2}$. Plasmids and siRNAs were transfected into HeLa cells using Lipofectamine 2000 (Thermo) and Lipofectamine RNAiMAX (Thermo), respectively. The detailed siRNA sequence and primers for mutagenesis are available in Supplementary Table 1.

The plasmids used for C.elegans RNAi were gifted by Prof. Lijun Kang (Zhejiang University). The mouse Dcaf13 coding sequence was amplified from an ovary cDNA mixture and was subcloned into pcDNA3.1-based Nterminal FLAG-tagged or GFP-tagged eukaryotic expression vectors. The cDNA sequence of FBL were cloned into pcDNA3.1(+)-mCherry vector by PCR amplification, enzyme digestion, and ligation. 


\section{In vitro mRNA synthesis and microinjection}

Plasmids were linearized by appropriate restriction enzymes. 5'-capped mRNAs were synthesized using Sp6 mMessage mMachine kit (Ambion), and poly (A) tails were added by a Poly (A) Tailing Kit (Ambion). The DNA template was removed by Turbo DNase treatment, and synthesized mRNA was extracted by phenol/chloroform extraction, followed by isopropanol precipitation. Microinjection was performed using a micromanipulator and microinjector (Eppendorf) under an inverted microscope (Eclipse TE200; Nikon). Synthetic mRNA (200 $\mu \mathrm{g} / \mathrm{ml})$ or siRNA $(20 \mu \mathrm{M})$ diluted in distilled water was microinjected into the cytoplasm of oocytes. Denuded oocytes were injected with $10 \mathrm{pL}$ samples per oocyte.

\section{Histologic analysis and IHC}

Ovaries were fixed overnight in 10\% PBS buffered formalin and then embedded in paraffin. Ovary samples were serially sectioned at $5 \mu \mathrm{m}$ thicknesses and were stained with hematoxylin and eosin. For IHC, sections were deparaffinized and rehydrated, and were incubated with primary antibodies for $1 \mathrm{~h}$ at room temperature, followed by biotinlabeled secondary antibodies for $30 \mathrm{~min}$. Staining procedure was performed using the Vectastain $\mathrm{ABC}$ kit and 3,3'diaminobenzidine peroxidase substrate kit (Vector Laboratories, Burlingame, California). The ovarian sections were counterstained with hematoxylin after IHC staining.

\section{Immunofluorescence}

Oocytes were fixed in $4 \%$ paraformaldehyde in PBS for $30 \mathrm{~min}$ at room temperature and permeabilized for $20 \mathrm{~min}$ in PBS containing $0.1 \%$ Triton X-100. After blocking with $1 \%$ BSA (bovine serum albumin) in PBST, oocytes were incubated with primary antibodies diluted in blocking solution at room temperature for $1 \mathrm{~h}$, then incubated with Alexa Fluor 594-conjugated or 488-conjugated secondary antibodies (Molecular Probes). Slides were mounted using VectaShield with 4',6-diamidino-2-phenylindole (DAPI, Vector Laboratories). Imaging of oocytes after immunofluorescence was performed on a Zeiss LSM710 confocal microscope. The antibodies used are listed in Supplementary Table 2.

\section{RNA isolation, reverse transcription (RT), and RT- PCR}

Total RNA of $C$. elegans was isolated using the TRIZOL reagent (Invitrogen) according to the manufacturer's instructions. Total RNA from oocytes was extracted using the RNeasy Mini kit (Qiagen) according to the manufacturer's instruction, followed by RT using Superscript RT kit (Bio-Rad). Quantitative RT-PCR was performed using a Power SYBR Green PCR Master Mix (Applied Biosystems, Life technologies) with ABI 7500 Real-Time PCR system (Applied Biosystems, Life technologies). Relative mRNA levels were calculated by normalizing to the levels of endogenous GAPDH mRNA (used as an internal control). The primers used are listed in Supplementary Table 1.

\section{Detection of transcription in oocytes}

Growing oocytes were incubated with $\alpha$-Amanitin $(10 \mu \mathrm{m})$ for $12 \mathrm{~h}$ or CX5461 $(10 \mu \mathrm{m})$ for $3 \mathrm{~h}$. Then the oocytes were transferred into a medium containing $1 \mathrm{mM} 5$-EU for $2 \mathrm{~h}$. EU staining was performed using Click-iT $^{\oplus}$ RNA Alexa Fluor 488 Imaging Kit (Life Technologies) according to manufacturer's instructions.

\section{Detection of protein synthesis}

Oocytes were incubated in M16 medium supplemented with $100 \mathrm{mM}$ HPG for $1 \mathrm{~h}$. Then the oocytes were fixed for 30 min at $37{ }^{\circ} \mathrm{C}$ in $3.7 \%$ formaldehyde. HPG was detected by Click-iT cell reaction kit (Life Technologies). The mean cytoplasmic signal was measured, crossed the middle of each oocyte, and quantified using ImageJ software.

\section{Fluorescence in situ hybridization}

FISH was performed with the antisense oligonucleotide probes listed in Supplementary Table 1. The probes were synthesized by Sango Biotech and labeled at the $5^{\prime}$ terminal end. The probes targeting $18 \mathrm{~S}$ and $28 \mathrm{~S}$ rRNAs was conjugated with Cy3 and Alexa Fluor 488, respectively.

Oocytes were fixed with $70 \%$ ethanol for $30 \mathrm{~min}$ at room temperature, washed in saline-sodium citrate buffer $(2 \times \mathrm{SSC}$, $0.3 \mathrm{M} \mathrm{NaCl}, 0.03 \mathrm{M} \mathrm{Na}_{3} \mathrm{C}_{6} \mathrm{H}_{5} \mathrm{O}_{7}, \mathrm{pH}$ 7.0) and placed into the hybridization mix solution. The hybridization mix contained $50 \%$ deionized formamide (Sigma-Aldrich), $10 \%$ dextran sulfate (Loba Feinchemie GMBH, Austria), 5\% $20 \times$ SSC ( $3 \mathrm{M} \mathrm{NaCl}, 0.3 \mathrm{M} \mathrm{Na}_{3} \mathrm{C}_{6} \mathrm{H}_{5} \mathrm{O}_{7}, \mathrm{pH} 7.0$ ), and $8 \mathrm{ng} / \mu \mathrm{l}$ probes. Hybridization was performed in a wet chamber for $18 \mathrm{~h}$ at $42{ }^{\circ} \mathrm{C}$. After hybridization, oocytes were sequentially washed with $50 \%$ formamide (Panreac, Spain) in $2 \times \operatorname{SSC}(3 \times$ $10 \mathrm{~min})$ at $42{ }^{\circ} \mathrm{C}, 2 \times \mathrm{SSC}$ at $42{ }^{\circ} \mathrm{C}(10 \mathrm{~min})$, and $2 \times \mathrm{SSC}$ (10 $\mathrm{min})$ at room temperature. The oocytes were counterstained with $1 \mu \mathrm{g} / \mathrm{ml}$ Hoechst 33342 for $15 \mathrm{~min}$ and mounted in Vectashield".

\section{Northern blot}

Total RNA was extracted from 100 oocytes using the RNeasy Mini kit (Qiagen) according to the manufacturer's instructions. The RNA probes were transcribed by T7 RNA polymerases 
and labeled with digoxigenin. Northern blot was performed following standard procedures of DIG Northern Starter Kit (Roche). The probes used are listed in Supplementary Table 1. The position of the probes was shown in Fig. S7B.

\section{RNA-binding protein immunoprecipitation}

HeLa cells were transiently transfected with Flag-DCAF13 for $48 \mathrm{~h}$. Cells were lysed in the lysis buffer $(50 \mathrm{mM}$ Tris- $\mathrm{HCl}$, pH 7.5, $150 \mathrm{mM} \mathrm{NaCl}, 10 \%$ glycerol, and $0.5 \%$ NP-40) for $30 \mathrm{~min}$ at $4{ }^{\circ} \mathrm{C}$. Protein-RNA complexes were immunoprecipitated at $4{ }^{\circ} \mathrm{C}$ using anti-Flag M2 beads (Sigma) and protein G Sepharose coated with anti-DCAF13 or protein $\mathrm{G}$ Sepharose coated with mouse $\mathrm{IgG}$ as the control. Beads were washed three times in the IP buffer. RNA was extracted with TRIZOL reagent (ThermoFisher Scientific).

\section{Western blot}

Oocytes or HeLa cells were lysed directly in $\beta$-mercaptoethanol containing loading buffer and heated at $95{ }^{\circ} \mathrm{C}$ for $5 \mathrm{~min}$. SDS-PAGE and immunoblots were performed following standard procedures using a Mini-PROTEAN Tetra Cell System (Bio-Rad, Hercules, CA). The antibodies used are listed in Supplementary Table 2.

\section{Polysome profile assay}

For polysome profile analysis, cells were cultured in $10-\mathrm{cm}$ dishes and transfected with non-targeting (siCtrl) or Dcafl3 siRNAs for $72 \mathrm{~h}$. Cells were washed with cold PBS with 100 $\mu \mathrm{g} / \mathrm{ml}$ cycloheximide and collected by centrifugation at 1000 $\mathrm{rpm}$ for $10 \mathrm{~min}$ at $4{ }^{\circ} \mathrm{C}$. Cell pellets were lysed in hypotonic lysis buffer [30 mmol/l Tris- $\mathrm{HCl}(\mathrm{pH} 7.5), 10 \mathrm{mmol} / \mathrm{l} \mathrm{MgCl}_{2}$, $100 \mathrm{mmol} / \mathrm{l} \mathrm{KCl}, 100 \mu \mathrm{g} / \mathrm{ml}$ cycloheximide, $1 \mathrm{mmol} / \mathrm{l} \mathrm{DTT}$, $0.25 \mathrm{mmol} / 1 \mathrm{Na}_{3} \mathrm{VO}_{4}, 1 \%$ Triton $\mathrm{X}-100$, and $0.5 \%$ sodium deoxycholate, protease inhibitor cocktail (EDTA-free)], and cellular debris was removed by centrifugation at $12,000 \times g$ for 15 min at $4{ }^{\circ} \mathrm{C}$. Lysates were loaded onto $15-50 \%$ sucrose density gradients $[30 \mathrm{mmol} / \mathrm{l}$ Tris- $\mathrm{HCl}(\mathrm{pH} 7.5), 100 \mathrm{mmol} / \mathrm{l}$ $\mathrm{NaCl}, 10 \mathrm{mmol} / \mathrm{l} \mathrm{MgCl}_{2}$, protease inhibitor cocktail (EDTAfree), and 100 units/ml RNase inhibitor] and centrifuged in a rotor at $34,000 \mathrm{rpm}$ for $2 \mathrm{~h}$ at $4{ }^{\circ} \mathrm{C}$. After centrifugation, gradients were fractionated at a rate of $2.5 \mathrm{ml} / \mathrm{min}$ ( 25 fractions, 12 drops per fraction) and the absorbance at $254 \mathrm{~nm}$ was continuously recorded (chart speed, $30 \mathrm{~cm} / \mathrm{h}$ ) using an ISCO fractionator (Teledyne ISCO).

\section{RNA-seq library preparation and gene expression level analysis}

Growing oocytes were collected from 14.5-day-old female mice. Fully grown stage oocytes were collected from 3- week-old mice after PMSG injection for $44 \mathrm{~h}$. Each sample (10 oocytes per sample) was added with $4 \mu$ lysis buffer (consisting of $0.2 \%$ TritonX-100, RNase inhibitor, dNTPs and oligo-dT primers) and immediately processed into cDNA using Smart-seq2 as described previously. Sequencing libraries were constructed from $500 \mathrm{pg}$ pre-amplified cDNA, using a DNA library prepration kit (TruePrep DNA Library Prep Kit V2 for Illumina, Vazyme), which was based on Tn5 transposase. Barcoded libraries were pooled at equimolar ratios and sequenced on illumina platform with 150 bp pair-end reads.

Sequencing reads were mapped against the reference genome (mm9) with Bowtie/TopHat version 2.0.14, which allowed mapping across splicing junctions by read segmentation. All programs were performed with default settings unless otherwise specified. The unique mapped reads (77\% of total reads) were subsequently assembled into transcripts guided by reference annotation (University of California at Santa Cruz [UCSC] gene models) with Cufflinks version 2.2.1. The expression level of each gene was quantified with normalized FPKM. Differentially expressed genes were identified by asking for a fold change $>2$ with FPKM $>1$ in at least one sample.

\section{Statistical analysis}

Results are given as means \pm SEM. Each experiment was repeated at least three times. Results for two experimental groups were compared by two-tailed unpaired Student's $t$ tests. Statistically significant values of $P<0.05, P<0.01$, and $P<$ 0.001 are indicated by asterisks $(*),(* *)$, and $(* * *)$, respectively. RNA-sequencing result statistical analyses were implemented with $\mathrm{R}$ (http://www.rproject.org). Independent twogroup Wilcoxon rank sum tests were used to compare distributions using the wilcox.test function in R. A Pearson's $R$ coefficient was calculated using the cor function with default parameters, and complete method was used to cluster the genes.

Acknowledgements This study is funded by the National Key Research and Developmental Program of China (2017YFC1001500, 2016YFC1000600, and 2017YFC1001100), National Natural Science Foundation of China (31528016, 31371449, and 31671558), and the Key Research and Development Program of Zhejiang Province (2017C03022).

\section{Compliance with ethical standards}

Conflict of interest The authors declare that they have no conflict of interest

\section{References}

1. Adhikari D, Liu K. Molecular mechanisms underlying the activation of mammalian primordial follicles. Endocr Rev. 2009;30:438-64. 
2. Liu K, Rajareddy S, Liu L, Jagarlamudi K, Boman K, Selstam G, et al. Control of mammalian oocyte growth and early follicular development by the oocyte PI3 kinase pathway: new roles for an old timer. Dev Biol. 2006;299:1-11.

3. Tan JH, Wang HL, Sun XS, Liu Y, Sui HS, Zhang J. Chromatin configurations in the germinal vesicle of mammalian oocytes. Mol Hum Reprod. 2009;15:1-9.

4. Yu C, Xu YW, Sha QQ, Fan HY. CRL4DCAF1 is required in activated oocytes for follicle maintenance and ovulation. Mol Hum Reprod. 2015;21:195-205.

5. Yu C, Zhang YL, Pan WW, Li XM, Wang ZW, Ge ZJ, et al. CRL4 complex regulates mammalian oocyte survival and reprogramming by activation of TET proteins. Science. 2013;342:1518-21.

6. Gu TP, Guo F, Yang H, Wu HP, Xu GF, Liu W, et al. The role of Tet3 DNA dioxygenase in epigenetic reprogramming by oocytes. Nature. 2011;477:606-10.

7. Lee J, Zhou P. DCAFs, the missing link of the CUL4-DDB1 ubiquitin ligase. Mol Cell. 2007;26:775-80.

8. Angers S, Li T, Yi X, MacCoss MJ, Moon RT, Zheng N. Molecular architecture and assembly of the DDB1-CUL4A ubiquitin ligase machinery. Nature. 2006;443:590-3.

9. Xu YW, Cao LR, Wang M, Xu Y, Wu X, Liu J, et al. Maternal DCAF2 is crucial for maintenance of genome stability during the first cell cycle in mice. J Cell Sci. 2017;130:3297-307.

10. Yu C, Ji SY, Sha QQ, Sun QY, Fan HY. CRL4-DCAF1 ubiquitin E3 ligase directs protein phosphatase 2A degradation to control oocyte meiotic maturation. Nat Commun. 2015;6:8017.

11. Zhang YL, Zhao LW, Zhang J, Le R, Ji SY, Chen C, et al. DCAF13 promotes pluripotency by negatively regulating SUV39H1 stability during early embryonic development. EMBO J. 2018;37:e98981
12. Zuccotti M, Giorgi Rossi P, Martinez A, Garagna S, Forabosco A, Redi CA. Meiotic and developmental competence of mouse antral oocytes. Biol Reprod. 1998;58:700-4.

13. Ma JY, Li M, Luo YB, Song S, Tian D, Yang J, et al. Maternal factors required for oocyte developmental competence in mice: transcriptome analysis of non-surrounded nucleolus (NSN) and surrounded nucleolus (SN) oocytes. Cell Cycle. 2013;12:1928-38

14. Bouniol-Baly C, Hamraoui L, Guibert J, Beaujean N, Szollosi MS, Debey P. Differential transcriptional activity associated with chromatin configuration in fully grown mouse germinal vesicle oocytes. Biol Reprod. 1999;60:580-7.

15. Kageyama S, Liu H, Kaneko N, Ooga M, Nagata M, Aoki F. Alterations in epigenetic modifications during oocyte growth in mice. Reproduction. 2007;133:85-94.

16. Lodde V, Modina S, Maddox-Hyttel P, Franciosi F, Lauria A, Luciano AM. Oocyte morphology and transcriptional silencing in relation to chromatin remodeling during the final phases of bovine oocyte growth. Mol Reprod Dev. 2008;75:915-24.

17. Rodriguez-Corona U, Sobol M, Rodriguez-Zapata LC, Hozak P, Castano E. Fibrillarin from Archaea to human. Biol Cell. 2015;107:159-74.

18. Duncan FE, Jasti S, Paulson A, Kelsh JM, Fegley B, Gerton JL. Age-associated dysregulation of protein metabolism in the mammalian oocyte. Aging Cell. 2017;16:1381-93.

19. Bax R, Vos HR, Raue HA, Vos JC. Saccharomyces cerevisiae Soflp associates with 35S Pre-rRNA independent from U3 snoRNA and Rrp5p. Eukaryot Cell. 2006;5:427-34.

20. Lan ZJ, Xu X, Cooney AJ. Differential oocyte-specific expression of Cre recombinase activity in GDF-9-iCre, Zp3cre, and Msx2Cre transgenic mice. Biol Reprod. 2004;71:1469-74. 